\title{
PERSISTENT MANIFOLDS ARE NORMALLY HYPERBOLIC
}

BY

\section{RICARDO MAÑÉ}

ABSTRACT. Let $M$ be a smooth manifold, $f: M \hookleftarrow$ a $C^{1}$ diffeomorphism and $V \subset M$ a $C^{1}$ compact submanifold without boundary invariant under $f$ (i.e. $f(V)=V)$. We say that $V$ is a persistent manifold for $f$ if there exists a compact neighborhood $U$ of $V$ such that $\bigcap_{n \in \mathbf{Z}} f^{n}(U)=V$, and for all diffeomorphisms $g$ : $M \hookleftarrow$ near to $f$ in the $C^{1}$ topology the set $V_{g}=$ $\bigcap_{n \in Z} g^{n} .(U)$ is a $C^{1}$ submanifold without boundary $C^{1}$ near to $V$. Several authors studied sufficient conditions for persistence of invariant manifolds. Hirsch, Pugh and Shub proved that normally hyperbolic manifolds are persistent, where normally hyperbolic means that there exist a $T f$-invariant splitting $T M / V=N^{s} V \oplus N^{u} V \oplus T V$ and constants $K>0,0<\lambda<1$ such that:

$$
\begin{gathered}
\left\|(T f)^{n} / N_{x}^{s} V\right\|<K \lambda^{n}, \quad\left\|(T f)^{-n} / N_{x}^{u} V\right\|<K \lambda^{n}, \\
\left\|(T f)^{n} / N_{x}^{s} V\right\| \cdot\left\|(T f)^{-n} / T_{f^{n}(x)} V\right\|<K \lambda^{n}
\end{gathered}
$$

for all $n>0, x \in V$. In this paper we prove the converse result, namely that persistent manifolds are normally hyperbolic.

Let $M$ be a manifold, $V \subset M$ a submanifold and $f: M \hookleftarrow$ a diffeomorphism satisfying $f(V) \subset V$. Several authors [1] [9] have considered under which conditions for every diffeomorphism $g$ nearby $f$ there exists a submanifold $V_{g} \subset M$ nearby $V$ satisfying $g\left(V_{g}\right) \subset V_{g}$. This kind of question arises frequently in ordinary, functional [18] and partial [16] differential equations as well as in the stability theory of group actions [19], bifurcations [3] and the construction of diffeomorphisms exhibiting certain persistent properties [9].

Answers to this problem are usually given in terms of exponential rates of the iterates of the derivative of $f$ at $V$. In this paper we shall consider the converse problem, proving that the persistence of $V$ (see definition below) is equivalent to these conditions.

To give the precise statement of the results let us denote by $\operatorname{Diff}^{1}(M)$ the space of $C^{1}$ diffeomorphisms of $M$ endowed with the topology of $C^{1}$ convergence on compact subsets.

Definition. $V$ is a compact invariant manifold of $f \in \operatorname{Diff}^{1}(M)$ if it is a compact boundaryless $C^{1}$ submanifold of $M$ satisfying $f(V)=V$. We say that $V$ is persistent if there exist a neighborhood $U$ of $V$ in $M$ and a

Received by the editors September 19, 1977.

AMS (MOS) subject classifications (1970). Primary 58F15; Secondary 58F10. 
neighborhood $थ$ of $f$ in $\operatorname{Diff}^{1}(M)$ such that:

(a) For all $g \in \mathcal{U}$ the set $V_{g}=\bigcap_{n \in \mathbf{Z}} g^{n}(U)$ is a $C^{1}$ submanifold of $M$ and $V_{g}=V$.

(b) $V_{g}$ is $C^{1}$ near to $V$ if $g$ is $C^{1}$ near to $f$.

Finally let us say that $V$ is normally hyperbolic if there exist constants $C>0,0<\lambda<1$ and a continuous (Tf)-invariant splitting $T M / V=N^{s} V$ $\oplus N^{u} V \oplus T V$ satisfying

$$
\begin{gathered}
\left\|(T f)^{n} / N_{x}^{s} V\right\| \leqslant C \lambda^{n}, \quad\left\|(T f)^{-n} / N_{x}^{u} V\right\| \leqslant C \lambda^{n}, \\
\left\|(T f)^{n} / N_{x}^{s} V\right\| \cdot\left\|(T f)^{-n} / T_{f^{n}(x)} V\right\| \leqslant C \lambda^{n}, \\
\left\|(T f)^{-n} / N_{x}^{u} V\right\| \cdot\left\|(T f)^{n} / T_{f^{-n}(x)} V\right\| \leqslant C \lambda^{n}
\end{gathered}
$$

for all $x \in \mathbf{Z}^{+}$.

In [9] it is proved that normal hyperbolicity implies persistence. Here we shall prove the converse, thus obtaining

THEOREM A. A compact invariant manifold of a diffeomorphism is persistent if and only if it is normally hyperbolic.

This theorem follows from a stronger version given in $\$ 2$ that states that a Lipschitz persistent compact invariant manifold (same definition as above requiring $V_{g}$ Lipschitz near to $V$ instead of $C^{1}$ near) is normally hyperbolic.

Recalling that a compact subset $\Lambda$ of a diffeomorphism $f \in \operatorname{Diff}^{l}(M)$ is said to be isolated if it has a neighborhood $U$ such that $\bigcap_{n \in Z} f^{n}(U)=\Lambda$, the Lipschitz version of Theorem A suggests the following conjecture:

Conjecture. There exists a residual subset $\mathscr{B} \subset \operatorname{Diff}^{1}(M)$ such that every isolated compact invariant manifold of a diffeomorphism in $\mathscr{B}$ is normally hyperbolic.

Hyperbolicity hypotheses (i.e. hypotheses involving exponential rates like normal hyperbolicity) are widely used in perturbation theory. However, the generalized feeling that they are good hypotheses in the sense that they are equivalent to the stability property they seek to grant, has, in terms of theorems, an incomplete justification. These problems when settled in $\operatorname{Diff}^{r}(M)$ with $r \geqslant 1$ are even harder for several reasons, among them the lack of a $C^{r}$ closing lemma [14]. This is, for instance, the main stumbling block to prove a $C^{r}$ version of Theorem A. Such a version would state a $C^{r}$ persistent invariant compact manifold (obvious definition) is $r$-normally hyperbolic in the sense defined in [9].

Finally I wish to thank Jacob Palis for his advice during the preparation of my thesis [10] (of which Theorem A is part) and to C. Pugh for several useful talks on Theorem A.

1. Domination and invariant subbundles. Let $K$ be a compact metric space. Denote by $\mathcal{L}(K)$ the set of continuous finite-dimensional vector bundles $F$ on 
$K$ endowed with a Riemann structure, i.e. a continuous map $B: F \times F \rightarrow \mathbf{R}$ such that $B /\left(F_{x} \times F_{x}\right)$ is an inner product on $F_{x}$ for all $x \in K$. If $F \in \mathcal{L}(K)$ let $\mathscr{L}(F)$ be the set of continuous vector bundle isomorphisms of $F$. If $\Phi \in \mathcal{L}(F)$ we say that a subbundle $E \subset F$ is $\Phi$-invariant if $\Phi(E)=E$. In the next proposition we give a sufficient condition for the existence of an invariant complement of $E$, i.e. a subbundle $G \subset F$ such that $G$ is $\Phi$-invariant and $\Phi(G)=G$. For the statement of the result we need the following definitions:

Definition 1.1. $F_{1}, F_{2} \in \mathcal{L}(K)$ and $\Phi_{i} \in \mathcal{L}\left(F_{i}\right), i=1,2$. We say that $\Phi_{1}$ dominates $\Phi_{2}$, denoted $\Phi_{1}>\Phi_{2}$, if there exist constants $C>0,0<\lambda<1$ such that

$$
\left\|\Phi_{1}^{n} / F_{1, x}\right\| \cdot\left\|\Phi_{2}^{-n} / F_{2, \Phi^{n}(x)}\right\| \leqslant C \lambda^{n}
$$

for all $x \in K, n \geqslant 0$. Moreover, if $\Phi_{1}>\Phi_{2}$ and (1) is satisfied we say that $\Phi_{1}$ $(C, \lambda)$-dominates $\Phi_{2}$.

Definition 1.2. Let $E$ be a finite-dimensional Hilbert space, $E_{1} \subset E$ a subspace and $E_{1}^{\perp}$ its orthogonal complement. If $S \subset E$ is a subspace such that $\operatorname{dim} S=\operatorname{dim} E_{1}$ and $S \cap E_{1}^{\perp}=\{0\}$, we define the angle $\alpha\left(E_{1}, S\right)$ by $\alpha\left(E_{1}, S\right)=\|L\|$, where $L: E_{1} \rightarrow E_{1}^{\perp}$ is a linear map satisfying $S=\operatorname{graph}(L)$.

Proposition 1.1. Let $F \in \mathfrak{L}(K), \Phi \in \mathcal{L}(F), E \subset F$ a continuous $\Phi$-invariant subbundle, $\tilde{F}=F / E$ the quotient bundle and $\tilde{\Phi} \in \mathcal{L}(\tilde{F})$ be the vector bundle isomorphism induced by $\Phi$.

If $\tilde{F}$ and $E$ have $\Phi$-invariant splittings $\tilde{F}=\tilde{F}_{1} \oplus \cdots \oplus \tilde{F}_{k}, E=E_{1}$ $\oplus \cdots \oplus E_{l}$ such that for all $1 \leqslant i \leqslant k, 1 \leqslant j \leqslant l$ either $\tilde{\Phi} / \tilde{F}_{i}(, \lambda)-$ dominates $\Phi / E_{j}$ or $\Phi / E_{j}(C, \lambda)$-dominates $\tilde{\Phi} / \tilde{F}_{i}$, then there exists a $\Phi$-invariant continuous subbundle $\hat{F} \subset F$ satisfying $\hat{F} \oplus E=F$ and such that for all $x \in K$,

where

$$
\alpha\left(E_{x}^{\perp}, \hat{F}_{x}\right) \leqslant \bar{C} d^{2} /(1-\lambda)
$$

$$
d=\sup \left\{\left\|\Phi / F_{x}\right\|,\left\|\Phi^{-1} / F_{x}\right\| \mid x \in K\right\}
$$

and $\bar{C}$ depends only on $C$ and

$$
\begin{gathered}
\delta=\sup \left\{\alpha\left(\left(\tilde{F}_{1} \oplus \cdots \oplus \tilde{F}_{j}\right)_{x}^{\perp},\left(\tilde{F}_{j+1} \oplus \cdots \oplus \tilde{F}_{k}\right)\right),\right. \\
\alpha\left(\left(E_{1} \oplus \cdots \oplus E_{1}\right)_{x}^{\perp},\left(E_{i+1} \oplus \cdots \oplus E_{l}\right)_{x}\right) \mid \\
1 \leqslant j<k, 1 \leqslant i<l, x \in K\} .
\end{gathered}
$$

REMARK. It follows from this proposition that if $\pi: \hat{F} \rightarrow \tilde{F}$ is the canonical projection and $\hat{F}_{j}=\pi^{-1} \tilde{F}_{j}, j=1, \ldots, k$, we have a $\Phi$-invariant continuous splitting $F=E \oplus \hat{F}_{1} \oplus \cdots \oplus \hat{F}_{j}$, and if $\tilde{\Phi} / \tilde{F}_{i}>\Phi / E_{j}\left(\Phi / E_{j}>\Phi / \tilde{F}_{i}\right)$ then $\Phi / \tilde{F}_{i}>\Phi / E_{j}\left(\Phi / E_{j}>\Phi / \hat{F}_{i}\right)$. 
Proof. Let $G$ be the continuous vector bundle on $K$ whose fiber on $x \in K$ is the space of linear transformations $L: E_{x}^{\perp} \rightarrow E_{x}$. Let $\Gamma^{0}(G)$ be the space of continuous sections of $G$ endowed with the norm $\|\eta\|=\{\|\eta(x)\| \mid x \in K\}$. Define $\hat{\Phi}: \Gamma^{0}(G) \hookleftarrow$ by

$$
\hat{\Phi}(\eta)(x)=\Phi \circ \eta\left(\Phi^{-1}(x)\right) \circ\left(\hat{\pi} \Phi / E_{\Phi^{-1}(x)}^{\perp}\right)^{-1}
$$

where $\hat{\pi}: F \rightarrow E^{\perp}$ is the orthogonal projection. Let $\tilde{\pi}: F \rightarrow \tilde{F}$ be the canonical projection. Define

$$
\hat{F}_{j}=E^{\perp} \cap \tilde{\pi}^{-1}\left(\tilde{F}_{j}\right), \quad 1 \leqslant j<k .
$$

Let $\Gamma^{s}$ (resp. $\Gamma^{u}$ ) be the space of sections $\eta \in \Gamma^{0}(G)$ such that $\eta(x) \hat{F}_{j, x} \subset$ $\bigoplus\left\{E_{i, x} \mid \Phi / E_{i}>\tilde{\Phi} / \tilde{F}_{j}\right\}\left(\eta(x) \hat{F}_{j, x} \subset \bigoplus\left\{E_{i, x} \mid \tilde{\Phi} / \tilde{F}_{j}>\Phi / E_{i}\right\}\right)$ for all $x \in K$. It is easy to see that

$$
\begin{gathered}
\left\|\hat{\Phi}^{n} / \Gamma^{s}\right\|<\hat{C} \lambda^{n}, \\
\left\|\hat{\Phi}^{-n} / \Gamma^{u}\right\| \leqslant \hat{C} \lambda^{n}
\end{gathered}
$$

for all $n \in \mathbf{Z}^{+}$. If we find $\eta_{0} \in \Gamma^{0}(G)$ satisfying

$$
\eta_{0}(x)=\hat{\Phi}\left(\eta_{0}\right)(x)+\pi \Phi \circ\left(\hat{\pi} \Phi / E_{\Phi^{-1}(x)}^{\perp}\right)^{-1}, \quad x \in K,
$$

where $\pi: F \rightarrow E$ is the orthogonal projection, then the subbundle $\hat{F} \subset F$ with fibers $\hat{F}_{x}=\operatorname{graph}\left(\eta_{0}(x)\right)$ is continuous and $\Phi$-invariant. To solve (3) observe that (1) and (2) imply (see [9] for details) that $I-\hat{\Phi}$ has an inverse that satisfies

$$
\left\|(I-\hat{\Phi})^{-1}\right\| \leqslant \frac{\bar{C}}{1-\lambda} .
$$

Let $\xi \in \Gamma^{0}(G)$ be defined by

$$
\xi(x)=\pi \Phi \circ\left(\hat{\pi} \Phi / E_{\Phi^{-1}(x)}^{\perp}\right)^{-1}, \quad x \in K .
$$

Then $\eta_{0}=(I-\hat{\Phi})^{-1} \xi$ satisfies (3) and

$$
\left\|\eta_{0}\right\| \leqslant(C /(1-\lambda))\|\xi\| \leqslant(C /(1-\lambda)) d^{2}
$$

where $d=\sup \left\{\left\|\Phi / F_{x}\right\|,\left\|\Phi^{-1} / F_{x}\right\| \mid x \in K\right\}$.

Proposition 1.2. Let $F \in \mathcal{L}(K), \Phi \in \mathcal{L}(F)$ and let $K_{0} \subset K$ be a $\Phi$-invariant compact subset. Suppose that $F / K_{0}$ has a continuous $\Phi$-invariant splitting $F / K_{0}=E_{0} \oplus F_{0}$ such that $\Phi / E_{0}>\Phi / F_{0}$. Then there exist a compact neighborhood $U$ of $K_{0}$ and a continuous subbundle $E \subset F / \Lambda^{s}$, where $\Lambda^{s}=\bigcap_{n>0} \Phi^{-n}(U)$, satisfying $\Phi(E) \subset E, E / K_{0}=E_{0}$.

Proof. Take a compact neighborhood $U_{0}$ of $K_{0}$ and let $\Lambda_{0}^{s}=$ $\cap_{n \in Z^{+}} \Phi^{-n}\left(U_{0}\right)$. If $U_{0}$ is small enough there exists a continuous splitting $F / \Lambda_{0}^{s}=\hat{E} \oplus \hat{F}$ such that $\hat{E} / K_{0}=E_{0}, \hat{F} / K_{0}=F_{0}$. Let $\pi_{1}: F / \Lambda_{0}^{s} \rightarrow \hat{E}, \pi_{2}$ : $F / \Lambda_{0}^{s} \rightarrow \hat{F}$ be the projections associated with this splitting. Let $G$ be the continuous vector bundle on $\Lambda_{0}^{s}$ whose fiber on $x$ is the space of linear maps 
$L: \hat{E}_{x} \rightarrow \hat{F}_{x}$. If $U \subset U_{0}$ is a compact neighborhood of $\Lambda_{0}^{s}$, let $\Gamma^{0}(U)$ be the space of continuous sections $\eta$ of $G / \Lambda^{s}(U)$, such that $\eta(x)=0$ if $x \in K_{0}$, where $\Lambda^{s}(U)=\bigcap_{n \in \mathbf{Z}^{+}} \Phi^{-n}(U)$, endowed with the norm $\|\eta\|=$ $\sup \left\{\|\eta(x)\| \mid x \in \Lambda^{s}(U)\right\}$. Define $\Phi_{1}: \hat{E} \hookleftarrow$ by $\Phi_{1}=\pi_{1} \Phi / \hat{E}, \Phi_{2}: \hat{F} \hookleftarrow$ by $\Phi_{2}=\pi_{2} \Phi / \hat{F}$ and $\hat{\Phi}: \Gamma^{0}(U) \hookleftarrow$ as $\hat{\Phi}(\eta)(x)=\Phi_{2}^{-1} \circ \eta(\Phi(x)) \circ \Phi_{1}$.

If $U$ is small enough $\hat{\Phi}$ is well defined.

LEMMA 1.1. There exist constants $C>0,0<\lambda<1$ and a neighborhood $U_{1} \subset U_{0}$ of $K_{0}$ such that for all compact neighborhoods $U \subset U_{1}$ of $K_{0}$ the linear map $\hat{\Phi}: \Gamma^{0}(U) \hookleftarrow$ satisfies $\left\|\hat{\Phi}^{n}\right\| \leqslant C \lambda^{n}$ for all $n \in \mathbf{Z}^{+}$.

Proof. There exists $n_{0} \in \mathbf{Z}^{+}$such that

$$
\left\|\Phi^{n_{0}} / E_{0, x}\right\| \cdot\left\|\Phi^{-n_{0}} / F_{0, \Phi^{n_{0}(x)}}\right\| \leqslant \frac{1}{3}
$$

for all $x \in K$. Then, there exists a neighborhood $U_{1}$ of $K_{0}$ such that

$$
\left\|\Phi_{1}^{n_{0}} / \hat{E}_{x}\right\| \cdot\left\|\Phi_{2}^{-n_{0}} / \hat{F}_{\Phi^{n_{0}}(x)}\right\|<\frac{1}{2}
$$

for all $x \in U_{1}$. Let $U \subset U_{1}$ be a compact neighborhood of $K_{0}$. If $x \in \Lambda^{s}(U)$, $\Phi^{n}(x) \in U$ for all $x \in \mathbf{Z}^{+}$. Therefore

$$
\left\|\Phi^{k n_{0}} / \hat{E}_{x}\right\| \cdot\left\|\Phi_{2}^{-k n_{0}} / F_{\Phi^{k n_{0}}(x)}\right\| \leqslant(1 / 2)^{k}
$$

for all $k \in \mathbf{Z}^{+}$. Then, for some constant $C>0,0<\lambda<1$ we have

$$
\left\|\Phi_{1}^{n} / \hat{E}_{x}\right\| \cdot\left\|\Phi_{2}^{-n} / \hat{F}_{\Phi^{n}(x)}\right\| \leqslant C \lambda^{n}
$$

for all $x \in \Lambda^{s}(U), n \in \mathbf{Z}^{+}$, and this implies

$$
\left\|\hat{\Phi}^{n}\right\| \leqslant C \lambda^{n}
$$

for all $n \in \mathbf{Z}^{+}$.

If we find $\eta_{0} \in \Gamma^{0}(U)$ such that $\Phi\left(\operatorname{graph}\left(\eta_{0}(x)\right)\right)=\operatorname{graph}\left(\eta_{0}(\Phi(x))\right)$ for all $x \in \Lambda^{s}(U)$, the lemma is proved, defining a subbundle $E$ of $F / \Lambda^{s}(U)$ by $E_{x}=\operatorname{graph}\left(\eta_{0}(x)\right)$. Let $\Gamma_{\varepsilon}^{0}(U)=\left\{\eta \in \Gamma^{0} \mid\|\eta\| \leqslant \varepsilon\right\}$. It is easy to see that there exist $\varepsilon_{2}>\varepsilon_{1}>0$ such that for all $\delta>0$ we can find a compact neighborhood $U_{\delta} \subset U$ of $K_{0}$ and a map $P: \Gamma_{\varepsilon_{1}}^{0}\left(U_{\delta}\right) \rightarrow \Gamma_{\varepsilon_{2}}^{0}\left(U_{\delta}\right)$ satisfying:

(a) $\Phi^{-1}(\operatorname{graph}(\eta(x)))=\operatorname{graph}\left(P(\eta)\left(\Phi^{-1}(x)\right)\right)$ for all $x \in \Phi\left(\Lambda^{s}\left(U_{\delta}\right)\right)$.

(b) $\|P(0)\| \leqslant \delta$.

(c) $\left\|(P-\hat{\Phi})\left(\eta_{1}\right)-(P-\hat{\Phi})\left(\eta_{2}\right)\right\| \leqslant \delta\left\|\eta_{1}-\eta_{2}\right\|$ for all $\eta_{1}, \eta_{2} \in \Gamma_{\varepsilon_{1}}^{0}\left(U_{\delta}\right)$.

Take $\delta \leqslant(1-\lambda) / 2 C\left(1+\varepsilon_{1}\right)$. From Lemma 1.1 it follows [4] that $I-\hat{\Phi}$ has an inverse satisfying $\left\|(I-\hat{\Phi})^{-1}\right\| \leqslant C /(1-\lambda)$. Moreover, if $\eta \in \Gamma_{\varepsilon_{1}}$ and $Q=P-\hat{\Phi}$,

$$
\begin{aligned}
&\left\|(I-\Phi)^{-1} Q(\eta)\right\| \\
& \leqslant\left\|(I-\Phi)^{-1} Q(\eta)-(I-\Phi)^{-1} Q(0)\right\|+\left\|(I-\Phi)^{-1} Q(0)\right\| \\
& \leqslant C \delta \varepsilon_{1} /(1-\lambda)+C \delta /(1-\lambda)<\frac{1}{2} \varepsilon_{2} ;
\end{aligned}
$$

hence, $(I-\Phi)^{-1} Q\left(\Gamma_{\varepsilon_{1}}^{0}\left(U_{\delta}\right)\right) \subset \Gamma_{\varepsilon_{2}}^{0}\left(U_{\delta}\right)$, and if $\eta_{1}, \eta_{2} \in \Gamma_{\varepsilon_{1}}^{0}\left(U_{\delta}\right)$ we have 


$$
\left\|(I-\Phi)^{-1} Q\left(\eta_{1}\right)-(I-\Phi)^{-1} Q\left(\eta_{2}\right)\right\| \leqslant \frac{C \delta}{1-\lambda}\left\|\eta_{1}-\eta_{2}\right\| \leqslant \frac{1}{2}\left\|\eta_{1}-\eta_{2}\right\| .
$$

By the fixed point theorem for contractions there exists $\eta_{0} \in \Gamma_{\varepsilon_{1}}^{0}\left(U_{\delta}\right)$ such that $(I-\hat{\Phi})^{-1} Q\left(\eta_{0}\right)=\eta_{0}$. Therefore $P\left(\eta_{0}\right)=\eta_{0}$ and, by $(\mathrm{a})$, the proposition is proved.

Proposition 1.3. Let $F \in \mathcal{L}(K), \Phi \in \mathcal{L}(F)$ and $K \subset K_{1}$ be a $\Phi$-invariant dense subset. Suppose that for all $x \in K_{1}$ there exists a splitting $F_{x}=E_{x} \oplus G_{x}$ satisfying:

(a) $\Phi\left(E_{x}\right)=E_{\Phi(x)}, \Phi\left(G_{x}\right)=G_{\Phi(x)}$.

(b) There exist $C>0,0<\lambda<1$ such that $\left\|\Phi^{n} / E_{x}\right\| \cdot\left\|\Phi^{-n} / G_{\Phi^{n}(x)}\right\| \leqslant$ $C \lambda^{n}$ for all $x \in \mathbf{Z}^{+}, x \in K_{1}$.

(c) $\operatorname{dim} E_{x}$ is constant.

Then the families of subspaces $\left\{E_{x} \mid x \in K\right\},\left\{G_{x} \mid x \in K\right\}$ extend to $\Phi$-invariant continuous subbundles $E$ and $G$ of $F$ such that $F=E \oplus G$ and $\Phi / E>\Phi / G$.

Proof. Let $\Sigma \subset K-K_{1}$ be a subset such that each $\Phi$-orbit contained in $K-K_{1}$ intersects $\Sigma$ at exactly one point. For each $x \in \Sigma$ chose a sequence $\left\{x_{n} \mid n \in \mathbf{Z}^{+}\right\} \subset K_{1}$ such that $x_{n} \rightarrow x$ when $n \rightarrow+\infty$ and the sequences $\left\{G_{x_{n}} \mid n \in \mathbf{Z}^{+}\right\},\left\{E_{x_{n}} \mid n \in \mathbf{Z}^{+}\right\}$are convergent. For $x \in \Sigma$ define

$$
G_{x}=\lim _{n \rightarrow+\infty} G_{x_{n}}, \quad E_{x}=\lim _{n \rightarrow+\infty} E_{x_{n}},
$$

and for each $x \in K-K_{1}$, choose $x_{k}=\Phi^{k}(x) \in \Sigma$ and define

$$
G_{x}=\Phi^{-k}\left(G_{x_{k}}\right), \quad E_{x}=\Phi^{-k}\left(E_{x_{k}}\right) \text {. }
$$

Let $P$ be the projective bundle associated to $F$ and $d(\cdot, \cdot)$ a metric on $P$ inducing its topology. If $A, B$ are subsets of $P$ define

$$
d(A, B)=\sup \{d(a, b) \mid a \in A, b \in B\} .
$$

Suppose that $\operatorname{dim} E_{x}=m, \operatorname{dim} G_{x}=n$ for all $x \in K$. Let $S \in \mathcal{L}(K)$ be the vector bundle whose fiber on $x$ is the set of subspaces $\left(L_{1}, L_{2}\right)$ of $F_{x}$ such that $\operatorname{dim} L_{1}=m, \operatorname{dim} L_{2}=n$. For $x \in K$ let $H_{x}$ be the set of $\left(L_{1}, L_{2}\right) \in S_{x}$ such that there exists a sequence $\left\{x_{n} \mid n \in \mathbf{Z}^{+}\right\} \subset K$ satisfying $x_{n} \rightarrow x, G_{x_{n}} \rightarrow L_{2}$, $E_{x_{n}} \rightarrow L_{1}$ when $n \rightarrow+\infty$. Proving that for all $x \in K, H_{x}=\left\{\left(E_{x}, G_{x}\right)\right\}$ we are done. Observe that $\cup_{x \in K} \tilde{H}_{x}$, where

$$
\tilde{H}_{x}=\left\{\theta \in P_{x} \mid \theta \subset L_{1} \cup L_{2},\left(L_{1}, L_{2}\right) \in H_{x}\right\}
$$

is a closed subset of $P$. Moreover, if $x \in K$, and $\left(L_{1}, L_{2}\right) \in H_{x}$ we have

$$
\left\|\Phi^{n} / L_{1}\right\| \cdot\left\|\Phi^{-n} / \Phi^{n}\left(L_{2}\right)\right\|=C \lambda^{n}
$$

for all $n \in Z^{+}$. In particular, $L_{1} \cap L_{2}=\{0\}$; hence $F_{x}=L_{1} \oplus L_{2}$. Let $x \in K,\left(L_{1}, L_{2}\right) \in H_{x}$ with $\left(L_{1}, L_{2}\right) \neq\left(E_{x}, G_{x}\right)$; let us say $L_{1} \neq E_{x}$. Then there exists $\theta \in P_{x} \cap L_{1}$ such that $\theta \cap E_{x}=\{0\}$. Since $\left(E_{x}, G_{x}\right) \in H_{x}$, (1) implies that $d\left(\Phi^{n}(\theta), G_{\Phi^{n}(x)}\right) \rightarrow 0$ when $n \rightarrow+\infty$. Let $y$ be an $\omega$-limit point of 
$x$. Take a sequence $\left\{n_{k} \mid k \in \mathbf{Z}^{+}\right\}$such that $\lim _{k \rightarrow+\infty} n_{k}=+\infty$ and the sequences $\Phi^{n_{k}}\left(L_{2}\right), \Phi^{n_{k}}\left(G_{x}\right), \Phi^{n_{k}}\left(E_{x}\right)$ converge to subspaces $L_{1}^{\prime}, L_{2}^{\prime}, S_{1}^{\prime}, S_{2}^{\prime}$ of $F_{x}$. Then $\left(L_{1}^{\prime}, L_{2}^{\prime}\right) \in H_{y},\left(S_{1}^{\prime}, S_{2}^{\prime}\right) \in H_{y}$ and $L_{1}^{\prime} \cap S_{2}^{\prime} \neq\{0\}$. Since $L_{1}^{\prime} \oplus L_{2}^{\prime}=$ $F_{y}$ we must have $L_{2}^{\prime} \not \subset S_{1}^{\prime}$. Take $\theta \in P_{y}$ such that $\theta \subset L_{2}^{\prime}$ and $\theta \cap S_{1}^{\prime} \neq\{0\}$. By (1) $d\left(\Phi^{-n}(\theta), \Phi^{-n}\left(S_{1}^{\prime}\right)\right) \rightarrow 0$ if $n \rightarrow+\infty$. Take an $\alpha$-limit point $z$ of $y$ and a sequence $\left\{n_{k} \mid k \in \mathbf{Z}^{+}\right\} \subset \mathbf{Z}^{+}$such that $\lim _{k \rightarrow+\infty} n_{k}=+\infty$ and $\Phi^{-n_{k}}\left(L_{1}^{\prime}\right)$, $\Phi^{-n_{k}}\left(L_{2}^{\prime}\right), \Phi^{-n_{k}}\left(S_{1}^{\prime}\right), \Phi^{-n_{k}}\left(S_{2}^{\prime}\right)$ converge to subspaces $L_{1}^{\prime \prime}, L_{2}^{\prime \prime}, S_{1}^{\prime \prime}, S_{2}^{\prime \prime}$. Then $\left(L_{1}^{\prime \prime}, L_{2}^{\prime \prime}\right) \in H_{z},\left(S_{1}^{\prime \prime}, S_{2}^{\prime \prime}\right) \in H_{z}, L_{2}^{\prime \prime} \cap S_{1}^{\prime \prime} \neq\{0\}$; take $0 \neq v_{1} \in L_{1}^{\prime \prime} \cap S_{2}^{\prime \prime}, 0$ $\neq v_{2} \in L_{2}^{\prime \prime} \cap S_{1}^{\prime \prime}$. Since $v_{1} \in L_{1}^{\prime \prime}, v_{2} \in L_{2}^{\prime \prime}$, (1) implies

$$
\left\|\Phi^{n}\left(v_{1}\right)\right\| /\left\|\Phi^{n}\left(v_{2}\right)\right\| \leqslant K \lambda^{n}\left(\left\|v_{1}\right\| /\left\|v_{2}\right\|\right)
$$

for all $n \in \mathbf{Z}^{+}$. But $v_{1} \in S_{2}, v_{2} \in S_{1}$. Hence (1) implies

$$
\left\|\Phi^{n}\left(v_{2}\right)\right\| /\left\|\Phi^{n}\left(v_{1}\right)\right\| \leqslant K \lambda^{n}\left(\left\|v_{2}\right\| /\left\|v_{1}\right\|\right),
$$

clearly contradicting (2).

2. Proof of Theorem A. Let $M$ be a $C^{\infty}$ boundaryless manifold and $V \subset M$ a $C^{1}$ compact boundaryless submanifold. Assume that $M$ is a submanifold of $\mathbf{R}^{n}$. Let $N V$ be a $C^{1}$ subbundle of $T M / V$ satisfying $T V \oplus N V=T M / V$. If $\eta$ is a section of $N V$ define the Lipschitz constant of $\eta$ by

$$
\operatorname{Lip}(\eta)=\sup \{\|\eta(x)-\eta(y)\| /\|x-y\| \mid x, y \in V, x \neq y\} .
$$

We say that $\eta$ is a Lipschitz section if $\operatorname{Lip}(\eta)<+\infty$. Let $\Gamma_{\mathrm{e}}(N V)$ be the space of Lipschitz sections of $N V$ endowed with the norm

$$
\|\eta\|_{\mathfrak{L}}=\sup \{\|\eta(x)\| \mid x \in V\}+\operatorname{Lip}(\eta) .
$$

Let $\operatorname{Diff}^{1}(M)$ be the space of $C^{1}$ diffeomorphisms with the topology of the $C^{1}$ convergence on compact subsets.

Definition 2.1. Let $f \in \operatorname{Diff}^{1}(M)$. We say that $V$ is a Lipschitz persistent invariant manifold of $f$ if there exists a neighborhood $U$ of $V$ such that for all $\delta>0$ there exists a neighborhood $\mathscr{Q}_{\delta}$ of $f$ such that if $g \in \mathcal{Q}_{\delta}$ there exists $\eta \in \Gamma_{\mathfrak{L}}(N V)$ with $\|\eta\|_{\mathfrak{L}} \leqslant \delta$ satisfying $V_{g}=\operatorname{graph}(\eta)$, where $\operatorname{graph}(\eta)=$ $\left\{\exp _{x}(\eta(x)) \mid x \in V\right\}, V_{g}=\bigcap_{n \in \mathbf{Z}^{+}} g^{n}(U)$.

Observe that this definition implies $V_{f}=V$, hence $f(V)=V$. Moreover, the Lipschitz persistence is independent of the bundle $N V$.

In this section we shall prove the following proposition, which clearly implies Theorem $\mathbf{A}$.

Proposition 2.1. If $V$ is a Lipschitz persistent invariant manifold of $f$ then $V$ is normally hyperbolic.

To prove this proposition we shall start showing some properties of the action of $T f$ on the Grassmannian bundle of $\operatorname{dim} V$-dimensional subspaces of the tangent space of $M$ at points of $V$. More precisely, let $S_{x}(M)$ be the set of $\operatorname{dim} V$-dimensional subspaces of $T_{x} M$. For $x \in V, S \in S_{x}(M)$ define $\alpha(S)$ 
$=\infty$ if $S \cap N_{x} V \neq\{0\}$ and $\alpha(S)=\|L\|$ if $S \cap N_{x} V=\{0\}$ and $L: T_{x} V \rightarrow$ $N_{x} V$ is the linear map satisfying $\operatorname{graph}(L)=S$. Let $\theta$ be the set of diffeomorphisms of $M$ that leave $V$ invariant.

LEMMA 2.1. For all $\delta>0$ there exist $\varepsilon=\varepsilon(\delta)$ and a neighborhood $Q=$ $\mathcal{U}(\delta)$ of $f$ such that if $g \in \mathcal{Q}, x \in V, S \in S_{x}(V), m \in \mathbf{Z}^{+}$and $\alpha(S)<\varepsilon$, $\alpha\left((T g)^{m} S\right) \leqslant \varepsilon$, then $\alpha\left((T g)^{j} S\right) \leqslant \delta$ for all $0 \leqslant j \leqslant m$.

Proof. Suppose the lemma false. Then there exists $\delta>0$ such that for all neighborhoods $\mathcal{Q}$ of $f$ and $\varepsilon>0$ we can find $y_{1} \in V, S \in S_{y_{1}}(V), g \in \mathcal{U} \cap$ $\vartheta$ and $0<n<m$ such that $\alpha(S)<\varepsilon, \alpha\left((T g)^{m} S\right)<\varepsilon$ and $\alpha\left(\left(T_{g}\right)^{n} S\right)>\delta$. We can assume without loss of generality that there exists a neighborhood $W_{1}$ of $y_{1}$ such that $W_{1} \cap g^{j}\left(W_{1}\right)=\varnothing$ for all $0<j<m$. Let $W_{2}=g^{m}\left(W_{1}\right)$. We can suppose that there exist $C^{\infty}$ diffeomorphisms $\varphi_{i}: W_{i} \rightarrow\left\{x \in \mathbf{R}^{l_{1}} \times\right.$ $\left.R^{l_{2}} \mid\|x\|<1\right\}, \quad i=1,2$, satisfying $\varphi_{i}\left(y_{i}\right)=0$ and $\varphi_{i}\left(W_{i} \cap V\right)=\{x \in$ $\left.\mathbf{R}^{l_{1}} \mid\|x\| \leqslant 1\right\}$. Take $L: \mathbf{R}^{l_{1}} \rightarrow \mathbf{R}^{l_{2}}, P: \mathbf{R}^{l_{1}} \rightarrow \mathbf{R}^{l_{2}}$ linear maps satisfying $\left(T \varphi_{1}\right) S$ $=\operatorname{graph}(L),\left(T \varphi_{2}\right)^{m} S=\operatorname{graph}(P)$. We can assume that $T \varphi_{i} / T_{y_{i}} M$ is an isometry for $i=1,2$. Hence $\|P\|<\varepsilon,\|L\|<\varepsilon$. Let $E_{1}$ be the kernel of $L$ and $E_{2}=E_{1}{ }^{\perp}$ its orthogonal complement. Take a $C^{\infty}$ function $\psi: \mathbf{R} \rightarrow \mathbf{R}$ satisfying $\psi(0)=1, \psi(t)=0$ for $|t|>1,\left|\psi^{\prime}(t)\right| \leqslant 2$, and $|\psi(t)|<1$ for all $t$.

Define: $B_{i}=\left\{x \in E_{i} \mid\|x\| \leqslant \frac{1}{2}\right\}, i=1,2, B_{3}=\left\{x \in R^{l_{2}} \mid\|x\| \leqslant \frac{1}{2}\right\} ; B$ $=B_{1} \times B_{2} \times B_{3}$. Let $F: B \rightarrow \mathbf{R}^{l_{2}}$ defined by

$$
F\left(x_{1}, x_{2}, x_{3}\right)=\psi\left(\left\|x_{1}\right\|^{2} / \lambda\right) \psi\left(\left\|x_{2}\right\|^{2} / \lambda\right) \psi\left(\left\|x_{3}\right\|^{2} / \lambda^{2}\right) L x_{2}
$$

where $\lambda$ is a positive number. Denoting by $F_{1}^{\prime}$ the partial derivative of $F$ with respect to the $i$ th variable, it is easy to see that

$$
\begin{gathered}
\sup _{B}\left\|F_{i}^{\prime}\right\| \rightarrow 0, \quad i=1,3, \\
\sup _{B}\left\|F_{2}^{\prime}\right\| \leqslant 2 \varepsilon, \\
\sup _{B}\|F\| \rightarrow 0
\end{gathered}
$$

when $\lambda^{-1} \rightarrow 0$. Now define $\varphi=\varphi_{2} \circ g^{m} \circ \varphi_{1}^{-1}, E_{1}^{\prime}=(T \varphi) E_{1}, E_{2}^{\prime}=(T \varphi) E_{2}$, $B_{i}^{\prime}=\left\{x \in E_{1} \mid\|x\| \leqslant 1\right\}, i=1,2, B^{\prime}=B_{1}^{\prime} \times B_{2}^{\prime}$. Take $\tilde{H}: B^{\prime} \rightarrow \mathbf{R}^{l_{2}}$ such that $\varphi\left(\operatorname{graph}\left(F / B_{1} \times B_{2}\right)\right)=\operatorname{graph}(\tilde{H})$. For small values, by $(1)-(3), \tilde{H}$ is well defined and satisfies

$$
\begin{aligned}
& \sup _{B^{\prime}}\left\|\tilde{H}_{1}^{\prime}\right\| \rightarrow 0, \\
& \sup _{B^{\prime}}\left\|\tilde{H}_{2}^{\prime}\right\| \leqslant \varepsilon, \\
& \sup _{B^{\prime}}\|\tilde{H}\| \rightarrow 0
\end{aligned}
$$

when $\quad \lambda \rightarrow 0$. Take $H: B^{\prime} \times B_{3} \rightarrow \mathbf{R}^{l_{2}}$ defined as $H\left(x_{1}, x_{2}, x_{3}\right)=$ $\psi\left(4 x_{3}\right) \tilde{H}\left(x_{1}, x_{2}\right)$. If $F^{\prime}, H^{\prime}$ denote the derivatives of $F$ and $H$ we can rewrite 
(4)-(6) as

$$
\begin{gathered}
\sup _{B}\left\|F^{\prime}\right\| \leqslant 2 \varepsilon, \\
\sup _{B^{\prime} \times B_{3}}\left\|H^{\prime}\right\| \leqslant 2 \varepsilon, \\
F^{\prime}(0) / B_{1} \times B_{2}=L, \quad H^{\prime}(0) / B^{\prime}=P .
\end{gathered}
$$

Take $k \in \operatorname{Diff}^{1}(M)$ defined by $k(x)=x$ if $x \notin W_{1} \cup W_{2}, k(x)=\left(\varphi_{1}^{-1} \circ(F\right.$ $\left.+I) \circ \varphi_{1}\right)(x)$ if $x \in W_{1}, k(x)=\left(\varphi_{2}^{-1} \circ(I-H) \circ \varphi_{2}\right)(x)$ for $x \in W_{2}$. Follows from (7) and (8) that $k$ is $C^{1}$ near to the identity if $\varepsilon$ and $\lambda$ are small. Hence, taking $g$ near enough to $f$ and $\varepsilon$ and $\lambda$ sufficiently small, it follows that $\bar{g}=k \circ g$ belongs to the neighborhood $U_{\delta}$ given by the definition of persistence. But observe that

$$
V_{\bar{g}}=\left(V-\bigcup_{0}^{m} \bar{g}^{j}\left(W_{1} \cap V\right)\right) \cup\left(\bigcup_{0}^{m} \bar{g}^{j}(D)\right)
$$

where $D=\varphi_{1}^{-1}(\operatorname{graph}(F))$. Then

$$
\alpha\left(T_{\bar{g}^{n}\left(y_{1}\right)} V_{\bar{g}}\right)=\alpha\left((T g)^{n} S\right)>\delta,
$$

thus contradicting the definition of $\mathscr{Q}_{\delta}$.

Now let $\tilde{N} V$ be the quotient bundle $(T M / V) / T V$ and $L(V)$ the vector bundle on $V$ whose fibers $L_{x}(V)$ are the spaces of linear transformations $L$ : $T_{x} V \rightarrow \tilde{N}_{x} V$. For $g \in \mathcal{O}$ define the vector bundle isomorphism $\Phi_{g}: L(V) \hookleftarrow$ by $\Phi_{g}(L)=\tilde{N} g \circ L \circ(T g)^{-1}$ where $\tilde{N} g: \tilde{N} V \hookleftarrow$ is the vector bundle isomorphism induced by $T g$. Let $\Phi=\Phi_{f}$.

LEMMA 2.2. $\Phi$ is a quasi-Anosov vector bundle isomorphism (i.e. [13] for all $0 \neq L \in L(V)$ the set $\left\{\left\|\Phi^{n}(L)\right\| \mid n \in \mathbf{Z}\right\}$ is unbounded $)$.

Proof. Suppose that $\Phi$ is not quasi-Anosov. Then there exist $\bar{x} \in V$ and $0 \neq L \in L_{\bar{x}}(V)$ such that $\left\{\left\|\Phi^{n}(L)\right\| \mid n \in \mathbf{Z}\right\}$ is bounded, let us say by $K>0$. Take a linear map $L_{0}: T_{\bar{x}} V \rightarrow N_{\bar{x}} V$ such that $\pi L_{0}=L$, where $\pi$ : $T M / V \rightarrow \tilde{N} V$ is the canonical projection. Let $S=\operatorname{graph}\left(L_{0}\right)$ and $\delta=\left\|L_{0}\right\|$ $=\alpha(S)$. Let $\mathcal{Q}(\delta / 2)$ and $\varepsilon(\delta / 2)$ be given by Lemma 2.1. As in [20, Lemma 1.1] we can find $1>\varepsilon_{0}>0$ such that given any finite subset $\Sigma \subset V$ and a family of linear maps $L_{x}: T_{x} M \rightarrow T_{f(x)} M, x \in \Sigma$, such that $L_{x}\left(T_{x} V\right)=T_{f(x)} V$ and $\left\|L_{x}-T f / T_{x} M\right\| \leqslant \varepsilon_{0}$ for all $x \in \Sigma$, then there exists $g \in \mathcal{Q}(\delta / 2)$ satisfying $g(V)=V, g(x)=f(x)$ and $T g / T_{x} M=L_{x}$ for all $x \in \Sigma$. Choose $n \in \mathbf{Z}^{+}$ satisfying

$$
\left(1-\frac{\varepsilon_{0}}{3}\right)^{n}<\frac{\varepsilon_{0} K^{-1} \varepsilon(\delta / 2)}{12 \operatorname{dim} V}
$$

and $\left(x, L^{\prime}\right)$ nearby $(\bar{x}, L)$ and $g \in \mathcal{O} \cap \mathcal{Q}(\delta / 3)$, such that $g^{j}(x) \neq g^{i}(x)$ for all $-n \leqslant j<i \leqslant n,\left\|\Phi_{g}^{j}\left(L^{\prime}\right)\right\| \leqslant K$ for all $|j|<n$ and $\left\|L_{0}^{\prime}\right\|>\delta / 2$ where 
$L_{0}^{\prime}: T_{x} V \rightarrow N_{x} V$ is a linear map satisfying $L^{\prime}=\pi L_{0}^{\prime}$. Let $S^{\prime}=\operatorname{graph}\left(L_{0}^{\prime}\right)$ and $\Sigma=\left\{g^{j}(x)|| j \mid \leqslant n\right\}$. By the definition of $\varepsilon_{0}$ there exists a map $g:\left[0, \varepsilon_{0} / 3\right] \rightarrow$ $\vartheta \cap \mathcal{Q}(\delta / 2)$ satisfying:

(1) $g_{0}=g$.

(2) $g_{\lambda}\left(g^{j}(x)\right)=g^{j+1}(x)$ for all $|j|<n$.

(3)

$$
\begin{array}{ll}
\tilde{N} g_{\lambda} / \tilde{N}_{g^{j}(x)} V=\left(1-\frac{\varepsilon_{0}}{3}\right) \tilde{N} g & \text { for all } 0 \leqslant j \leqslant n, \\
\left(N g_{\lambda}\right)^{-1} / N_{g^{j}(x)} V=\left(1-\frac{\varepsilon_{0}}{3}\right)(N g)^{-2} & \text { for all }-n \leqslant j \leqslant 0 .
\end{array}
$$

(4)

$$
\begin{gathered}
\left(T g_{\lambda}\right)^{n} / T_{x} V=(1+\lambda)(T g)^{n}, \quad\left(T g_{\lambda}\right)^{n} / T_{x} V=(1+\lambda)(T g)^{n}, \\
\left(T g_{\lambda}\right)^{-n} / T_{x} V=(1+\lambda)(T g)^{-n}
\end{gathered}
$$

We claim that for some $\lambda_{0} \in\left[0, \varepsilon_{0} / 3\right]$ we have $\alpha\left(\left(T g_{\lambda_{0}}\right)^{-n} S^{\prime}\right)<\varepsilon(\delta / 2)$ and $\alpha\left(\left(T g_{\lambda_{0}}\right)^{n} S^{\prime}\right) \leqslant \varepsilon(\delta / 2)$. Since $\alpha\left(S^{\prime}\right)>\delta / 2$ this contradicts $g_{\lambda_{0}} \in \mathcal{Q}(\delta / 2) \cap$ $\theta$ and the lemma is proved. To find $\lambda_{0}$ we shall use the following property: Let $L_{\lambda}^{+}: T_{g^{n}(x)} V \rightarrow N_{g^{n}(x)} V, L_{\lambda}^{-}: T_{g^{n}(x)} V \rightarrow N_{g^{-n}(x)} V$ be linear maps such that $\operatorname{graph}\left(L_{\lambda}^{+}\right)=\left(T g_{\lambda}\right)^{n} S^{\prime}, \operatorname{graph}\left(L_{\lambda}^{-}\right)=\left(T g_{\lambda}\right)^{-{ }^{n} S^{\prime}}$ and let $\left\{v_{1}^{+}, \ldots, v_{I}^{+}\right\}$, $\left\{v_{1}^{-}, \ldots, v_{\lambda}^{-}\right\}$be orthonormal bases of $T_{g^{n}(x)} V$ and $T_{g^{-n}(x)} V$. Then if $\bar{\lambda} \in$ $\left[0, \varepsilon_{0} / 3\right], \quad 1 \leqslant j \leqslant l$ and $\left\|L_{\lambda}^{ \pm} v_{j}^{+}\right\| \geqslant \varepsilon(\delta / 2)$ (resp. $\left\|L_{\bar{\lambda}}^{-} v_{j}^{-}\right\| \geqslant \varepsilon(\delta / 2)$ ), it follows that $\left\|L_{\lambda}^{+} v_{j}^{+}\right\| \leqslant \varepsilon(\delta / 2)\left(\left\|L_{\lambda}^{-} v_{j}^{-}\right\| \leqslant \varepsilon(\delta / 2)\right)$ for all $\bar{\lambda}+\varepsilon_{0} / 6 \operatorname{dim} V$ $\leqslant \lambda \leqslant \varepsilon_{0} / 3$. With this property it is easy to find the desired $\lambda_{0}$ as follows: Suppose that for some $j$ we have $\left\|L_{0}^{+} v_{j}^{+}\right\| \leqslant \varepsilon(\delta / 2)$. Then $\left\|L_{\lambda_{1}}^{+} v_{j}^{+}\right\|<\varepsilon(\delta / 2)$ where $\lambda_{1}=\varepsilon_{0} / 6 \operatorname{dim} V$. Now suppose that $\left\|L_{\lambda_{1}}^{+} v_{i}^{+}\right\| \geqslant \varepsilon(\delta / 2)$. Then $\left\|L_{2 \lambda}^{+} v_{i}^{+}\right\|$ $\leqslant \varepsilon(\delta / 2)$ and $\left\|L_{2 \lambda_{1}}^{+} v_{j}^{+}\right\| \leqslant \varepsilon(\delta / 2)$. At the end of this method we find $\lambda_{0}=$ $m \lambda_{1}$, with $0<m<2 \operatorname{dim} V$ (so $\lambda_{0} \in\left[0, \varepsilon_{0} / 3\right]$ ). It remains to prove the property above. For this define the linear maps $A_{\lambda}: N_{x} V \rightarrow N_{f^{n}(x)} V, P_{\lambda}$ : $N_{x} V \rightarrow T_{f^{n}(x)} V, Q_{\lambda}: T_{x} V \rightarrow T_{f^{n}(x)} V$ such that

$$
\left(T g_{\lambda}\right)^{n} / T_{x} M=\left(\begin{array}{cc}
A_{\lambda} & P_{\lambda} \\
0 & Q_{\lambda}
\end{array}\right)
$$

Then, if $w_{j}=Q_{0}^{-1} v_{j}^{+}$,

$$
\left\|L_{\lambda}^{+} v_{j}^{+}\right\|=\frac{\left\|A_{\lambda} L_{0}^{\prime} w_{j}\right\|}{\left\|Q_{0}(1+\lambda) w_{j}+P_{\lambda} L_{0}^{\prime} w_{j}\right\|}=\frac{\left\|\left(1-\varepsilon_{0} / 3\right)^{n} A_{0} L_{0}^{\prime} w_{j}\right\|}{\left\|Q_{0}(1+\lambda) w_{j}+P_{0} L_{0}^{\prime} w_{j}\right\|} .
$$

Suppose $\left\|L_{\lambda}^{+} v_{j}^{+}\right\| \geqslant \varepsilon(\delta / 2)$. Then, if $\lambda>\bar{\lambda}+\varepsilon_{0} / 6 \operatorname{dim} V$,

$$
\left\|Q_{0}(1+\lambda) w_{j}+P_{0} L_{0}^{\prime} w_{j}\right\| \geqslant(\lambda-\bar{\lambda})\left\|Q_{0} w_{j}\right\|-\left\|Q_{0}(1+\bar{\lambda}) w_{j}+P_{0} L_{0}^{\prime} w_{j}\right\| ;
$$

hence, 


$$
\begin{aligned}
\left\|L_{\lambda}^{+} v_{j}^{+}\right\| & \leqslant \frac{1}{\frac{\lambda-\bar{\lambda}}{\left(1-\varepsilon_{0} / 3\right)^{n}} \cdot \frac{\left\|Q_{0} w_{j}\right\|}{\left\|A_{0} L_{0}^{\prime} w_{j}\right\|}-\frac{1}{\left\|L_{\lambda}^{+} v_{j}\right\|}} \\
& \leqslant \frac{1}{\frac{\lambda-\bar{\lambda}}{\left(1-\varepsilon_{0} / 3\right)^{n}} K^{-1}-(\varepsilon(\delta / 2))^{-1}} .
\end{aligned}
$$

By (9),

$$
\frac{\lambda-\bar{\lambda}}{\left(1-\varepsilon_{0} / 3\right)^{n}} K^{-1} \geqslant \frac{\varepsilon_{0} K^{-1}}{6 \operatorname{dim} V\left(1-\varepsilon_{0} / 3\right)^{n}} \geqslant 2(\varepsilon(\delta / 2))^{-1}
$$

hence (10) implies

$$
\left\|L_{\lambda}^{+} v_{j}^{+}\right\| \leqslant \varepsilon(\delta / 2) \text {. }
$$

In the next lemma we show that $T M / \Omega(f / V)$ (where $\Omega(f / V)$ denotes the set of nonwandering points of $f / V$ ) has a splitting satisfying the domination conditions required by the definition of normal hyperbolicity.

LEMMA 2.3. There exists a continuous Tf-invariant splitting $T M / \Omega(f / V)=$ $T V / \Omega(f / V) \oplus N^{s} \oplus N^{u}$ such that $T f / N^{s}>T f / T V>T f / N^{u}$.

Proof. If $g \in \mathcal{O}$ define $E_{x}^{s}(g)$ (resp. $\left.E_{x}^{u}(g)\right)$ as the set of $L \in L_{x}(V)$ such that $\left\{\left\|\Phi_{g}^{n}(L)\right\| \mid n \geqslant 0\right\}\left(\left\{\left\|\Phi_{g}^{-n}(L)\right\| \mid n \geqslant 0\right\}\right)$ is bounded,

$$
\begin{aligned}
\tilde{N}_{x}^{s}(g) & =\bigcup\left\{L\left(T_{x} V\right) \mid L \in E_{x}^{s}(g)\right\}, \\
N_{x}^{u}(g) & =\cup\left\{L\left(T_{x} V\right) \mid L \in E_{x}^{u}(g)\right\}
\end{aligned}
$$

and $\operatorname{Per}(g)$ as the set of periodic points of $g / V$. In the proof of this lemma we shall use the following property whose proof will be given later:

LEMMA 2.4. There exist a neighborhood $\mathcal{Q}$ of $f$ and a dense subset $\mathfrak{S}$ of थ $\cap \mathcal{O}$ such that for all $g \in \mathcal{S}$ and $x \in \operatorname{Per}(g)$ we have $\tilde{N}_{x}^{s}(g) \cap \tilde{N}_{x}^{u}(g)=$ $\{0\}$.

Suppose we show that

$$
\tilde{N}_{x}^{s}(f) \oplus \tilde{N}_{x}^{u}(f)=\tilde{N}_{x} V
$$

for all $x \in \Omega(f / V)$ and that there exist $C>0,0<\lambda<1$ satisfying

$$
\begin{aligned}
& \left\|(\tilde{N} f)^{n} / \tilde{N}_{x}^{s}(f)\right\| \cdot\left\|(T f)^{-n} / T_{f^{n}(x)} V\right\|<C \lambda^{n}, \\
& \left\|(\tilde{N} f)^{-n} / \tilde{N}_{x}^{u}(f)\right\| \cdot\left\|(T f)^{n} / T_{f^{-n}(x)} V\right\|<C \lambda^{n}
\end{aligned}
$$

for all $x \in \Omega(f / V), n>0$. Then by Proposition 1.1 the maps $x \rightarrow \tilde{N}_{x}^{s}(f)$, $x \rightarrow \tilde{N}_{x}^{u}(f)$ define continuous $\tilde{N} f$-invariant subbundles $\tilde{N}^{s}, \tilde{N}^{u}$ of $\tilde{N} V / \Omega(f / V)$ such that $\tilde{N} f / N^{s}>T f /(T V / \Omega(f / V))>\tilde{N} f / N^{u}$. Hence, by Lemma 1.1, there exists a continuous $T$ f-invariant splitting satisfying our statement. So if we prove (11)-(13) we are done. By [13, Proposition 1.1 and 
Lemma 1.2] there exist a neighborhood $\mathcal{Q}$ of $f, C>0$ and $0<\lambda<1$ such that $\Phi_{g}$ is quasi-Anosov and

$$
\begin{gathered}
\left\|\Phi_{g}^{n} / E_{x}^{s}(g)\right\|<K \lambda^{n}, \\
\left\|\Phi_{g}^{-n} / E_{x}^{u}(g)\right\|<K \lambda^{n}
\end{gathered}
$$

for all $g \in \mathcal{Q} \cap \mathcal{\theta}, x \in V$ and $n \geqslant 0$. Moreover, since $\Phi_{g}$ is quasi-Anosov, it is easy to see that $E_{x}^{s}(g) \oplus E_{x}^{u}(g)=L_{x}(V)$, hence $\tilde{N}_{x}^{s}(g)+\tilde{N}_{x}^{u}(g)=\tilde{N}_{x} V$ for all $g \in \mathcal{Q} \cap \mathcal{O}, x \in \operatorname{Per}(g)$. Let $G_{x}^{s}(g), G_{x}^{u}(g)$ be the spaces of linear maps from $T_{x} V$ into $\tilde{N}_{x}^{s}(g)$ and $\tilde{N}_{x}^{u}(g)$, respectively. We claim that $g \in \mathcal{Q} \cap$ $\delta$ and $x \in \operatorname{Per}(g)$ imply

$$
\begin{aligned}
& G_{x}^{s}(g)=E_{x}^{s}(g), \\
& G_{x}^{u}(g)=E_{x}^{u}(g)
\end{aligned}
$$

Suppose, for instance, that (16) is false. Then $\operatorname{dim} G_{x}^{s}(g)>\operatorname{dim} E_{x}^{s}(g)$. Therefore,

$$
\begin{aligned}
\operatorname{dim} T_{x} V \cdot \operatorname{dim} \tilde{N}_{x} V & =\operatorname{dim} L_{x}(V)=\operatorname{dim} E_{x}^{s}(g)+\operatorname{dim} E_{x}^{u}(g) \\
& <\operatorname{dim} G_{x}^{s}(g)+\operatorname{dim} G_{x}^{u}(g) \\
& =\operatorname{dim} T_{x} V\left(\operatorname{dim} \tilde{N}_{x}^{s}(g)\right)+\operatorname{dim} \tilde{N}_{x}^{u}(g) \\
& =\operatorname{dim} T_{x} V \cdot \operatorname{dim} \tilde{N}_{x} V .
\end{aligned}
$$

Now consider $x \in \Omega(f / V)$. There exist sequences $\left\{g_{n} \mid n \in \mathbf{Z}^{+}\right\} \subset \delta,\left\{x_{n} \mid n\right.$ $\left.\in \mathbf{Z}^{+}\right\} \subset V$ such that $x_{n} \in P\left(g_{n}\right), g_{n} \rightarrow g$ and $x_{n} \rightarrow x$ when $n \rightarrow+\infty$ [14]. We can assume that there exist subspaces $E^{+}, E^{-}$of $L_{x}(V)$ such that

$$
\begin{aligned}
& \lim E_{x_{n}}^{s}\left(g_{n}\right)=E^{+}, \\
& \lim E_{x_{n}}^{u}\left(g_{n}\right)=E^{-} .
\end{aligned}
$$

Since $E_{x_{n}}^{s}\left(g_{n}\right) \oplus E_{x_{n}}^{u}\left(g_{n}\right)=L_{x}(V)$ we obtain

$$
\operatorname{dim} E^{+}+\operatorname{dim} E^{-}=\operatorname{dim} L_{x}(V)
$$

and (14), (15) imply $\left\|\Phi^{n} / E^{+}\right\|<K \lambda^{n}$, $\left\|\Phi^{-n} / E^{-}\right\| \leqslant K \lambda^{n}$ for all $n>0$. Since $\Phi$ is quasi-Anosov this implies $E^{+} \cap E^{-}=\{0\}$, which together with (20) gives $L_{x}(V)=E^{+} \oplus E^{-}$, hence $E^{+}=E_{x}^{s}(f), E^{-}=E_{x}^{u}(f)$; then

$$
E_{x}^{s}(f) \oplus E_{x}^{u}(f)=L_{x}(V) .
$$

Moreover, (16)-(19) and the elementary properties

$$
G_{x}^{s}(f)=\lim G_{x_{n}}^{s}\left(g_{n}\right), \quad G_{x}^{u}(f)=\lim G_{x_{n}}^{u}\left(g_{n}\right)
$$

prove that

$$
\begin{aligned}
& G_{x}^{s}(f)=E_{x}^{s}(f), \\
& G_{x}^{u}(f)=E_{x}^{u}(f) .
\end{aligned}
$$

By (21) $\tilde{N}_{x}^{s}(f)+\tilde{N}_{x}^{u}(f)=\tilde{N}_{x} V$ and by $(21)-(23), \tilde{N}_{x}^{s}(f) \cap \tilde{N}_{x}^{u}(f)=\{0\}$. This proves (11). (12) and (13) follow from (14), (15), (22) and (23). 
Proof of Lemma 2.4. Let $\mathcal{U}$ be a neighborhood of $f$ such that $\Phi_{g}$ is quasi-Anosov for all $g \in \mathcal{O} \cap \mathcal{U}$. Let $\mathcal{S}$ be the set of diffeomorphisms $g \in \mathcal{U} \cap \mathcal{O}$ satisfying the following properties:

(I) $\tilde{N}_{g}$ is a $C^{1}$ vector bundle isomorphism.

(II) $\operatorname{Per}(g)$ is dense in $\Omega(g / V)$.

(III) Every periodic point of $g / V$ is hyperbolic.

(IV) If $x \in \operatorname{Per}(g)$ and $m$ is its period, all the eigenvalues of $(T f)^{m} / T_{x} M$ are simple and two of them have equal modulus if and only if they are conjugates.

Now observe that for all $g \in \mathcal{S}$, the set of points $x \in \operatorname{Per}(g)$ such that $\tilde{N}_{x}^{s}(g) \cap \tilde{N}_{x}^{u}(g)=\{0\}$ is closed in $\operatorname{Per}(g)$. To see this suppose that $\left\{x_{n} \mid n \in\right.$ $\left.\mathbf{Z}^{+}\right\}$is a sequence contained in $\operatorname{Per}(g), g \in \mathcal{S}$, and $x_{n} \rightarrow x \in \operatorname{Per}(g)$ when $n \rightarrow+\infty$. If $\tilde{N}_{x_{n}}^{s}\left(g_{n}\right) \cap \tilde{N}_{x_{n}}^{u}\left(g_{n}\right)=\{0\}$, we deduce, as in the proof of Lemma 2.3, that $G_{x_{n}}^{s}(g)=E_{x_{n}}^{s}(g), G_{x_{n}}^{u}(g)=E_{x_{n}}^{u}(g)$ and

$$
\lim E_{x_{n}}^{s}\left(g_{n}\right)=E_{x}^{s}(g), \quad \lim E_{x_{n}}^{u}\left(g_{n}\right)=E_{x}^{u}(g) .
$$

Since, clearly, $\lim G_{x_{n}}^{s}(g)=G_{x}^{s}(g)$ and $\lim G_{x_{n}}^{u}(g)=G_{x}^{u}(g)$, it follows that $E_{x}^{s}(g)=G_{x}^{s}(g), E_{x}^{u}(g)=G_{x}^{u}(g)$. If $\tilde{N}_{x}^{s}(g) \cap \tilde{N}_{x}^{u}(g) \neq\{0\}$, these relations imply $E_{x}^{s}(g) \cap E_{x}^{u}(g) \neq\{0\}$, contradicting the fact that $\Phi_{g}$ is quasi-Anosov. Suppose then that for some $g_{0} \in \mathcal{S}$ there exists $a \in \operatorname{Per}\left(g_{0}\right)$ satisfying $\tilde{N}_{a}^{s}(g)$ $\cap \tilde{N}_{a}^{u}(g) \neq\{0\}$. By the previous remark this implies that there exists an open subset $W$ of $\operatorname{Per}\left(g_{0}\right)$ such that $\tilde{N}_{x}^{s}\left(g_{0}\right) \cap \tilde{N}_{x}^{u}\left(g_{0}\right) \neq\{0\}$ for every $x \in W$. To exhibit a contradiction between this fact and our definition of $\mathcal{S}$ we need the following definitions and Lemmas 2.5-2.7.

Definition 2.2. Let $g \in \mathcal{S}, x \in \operatorname{Per}(g)$. We say that

$$
\begin{gathered}
\tilde{N}_{x} V=\tilde{N}_{1} \oplus \cdots \oplus \tilde{N}_{l}, \\
T_{x} V=T_{1} \oplus \cdots \oplus T_{k}
\end{gathered}
$$

are the canonical splittings at $x$ if they satisfy the following conditions:

(I) $(T g)^{m} T_{i}=T_{i},(\tilde{N} g)^{m} \tilde{N}_{j}=\tilde{N}_{j}$ for all $1<i \leqslant k, 1<j<l$, where $m$ is the period of $x$.

(II) $(T g)^{m} / T_{i_{1}}>(T g)^{m} / T_{i_{2}},(\tilde{N} g)^{m} / \tilde{N}_{j_{1}}>(\tilde{N} g)^{m} / \tilde{N}_{j_{2}}$ for all $1<i_{1}<i_{2}<$ $k, 1 \leqslant j_{1}<j_{2} \leqslant l$.

(III) For all $1 \leqslant i \leqslant k, 1 \leqslant j \leqslant l,(\tilde{N} g)^{m} / \tilde{N}_{j}>(T g)^{m} / T_{i}$ or $(T g)^{m} / T_{i}>$ $(\tilde{N} g)^{m} / \tilde{N}_{j}$.

(IV) For all $1 \leqslant i_{1}<i_{2} \leqslant k$ there exists $1 \leqslant j<l$ such that $(T g)^{m} / T_{i_{1}}>$ $(\tilde{N} g)^{m} / \tilde{N}_{j}>(T g)^{m} / T_{i_{2}}$.

LEMMa 2.5. There exists $\gamma>0$ such that if $g \in \mathcal{S}, x \in \operatorname{Per}(g)$ and (24), (25) are the canonical splittings at $x$, then

$$
\begin{aligned}
& \alpha\left(\left(T_{1} \oplus \cdots \oplus T_{i}\right)^{\perp},\left(T_{i+1} \oplus \cdots \oplus T_{k}\right)\right)<\gamma, \\
& \alpha\left(\left(\tilde{N}_{1} \oplus \cdots \oplus \tilde{N}_{j}\right)^{\perp},\left(\tilde{N}_{j+1} \oplus \cdots \oplus \tilde{N}_{l}\right)\right)<\gamma
\end{aligned}
$$

for all $1<i<l, 1 \leqslant j<l$. 
Proof. We shall prove only the first inequality. The second one is proved applying the same method. From (14) and (15) it follows that there exists $k>0$ such that

$$
\left\|L_{1}-L_{2}\right\| \geqslant k
$$

for all $g \in \mathcal{Q}, x \in V, L_{1} \in E_{x}^{s}(g), L_{2} \in E_{x}^{u}(g),\left\|L_{1}\right\|=\left\|L_{2}\right\|=1$. Suppose that, contradicting the lemma, we can find for $\varepsilon=k / 3$ a diffeomorphism $g \in \delta$ and $x \in \operatorname{Per}(g)$ with canonical splittings (24), (25) such that $\alpha\left(T_{-}^{\perp}\right.$, $\left.T_{+}\right) \leqslant \varepsilon$, where $T_{+}=T_{i+1} \oplus \cdots \oplus T_{k}, T_{-}=T_{1} \oplus \cdots \oplus T_{i}$. Suppose, for instance, that $\operatorname{dim} T_{-}^{\perp} \leqslant \operatorname{dim} T_{+}$. Let $T^{\prime} \subset T_{+}, T^{\prime \prime} \subset T_{+}$be subspaces such that $\operatorname{dim} T^{\prime}=\operatorname{dim} T_{-}, T^{\prime \prime}=\left(T^{\prime} \oplus T_{-}\right)^{\perp}$. Take an isometry $G$ : $T_{x} V \hookleftarrow$ such that $\|G-I\| \leqslant 2 \varepsilon, G\left(T^{\prime \prime}\right)=T^{\prime \prime}, G\left(T^{\prime}\right)=T_{-}$. Let $1 \leqslant j \leqslant l$ satisfy $^{\prime}(T g)^{m} / T_{-}>(\tilde{N} g)^{m} \tilde{N}_{j}>(T g)^{m} / T_{+}$and $L: T_{x} V \rightarrow \tilde{N}_{j}$ be a linear map with $\|L\|=1, L / T_{+}=0, L G / T^{\prime} \neq 0$. Then $L \in E_{x}^{u}(g), L G \in E_{x}^{s}(g)$ and $\|L-G\| \leqslant\|L\| \cdot\|I-G\| \leqslant 2 \varepsilon=\frac{2}{3} k$, contradicting (26).

Lemma 2.6. There exist $C^{\prime}>0,0<\lambda<1$ such that if $g \in \mathcal{S}, x \in \operatorname{Per}(g)$ and (24), (25) are the canonical splittings at $x$, then for all $1<j<l, 1<i<l$ we have

$$
\left\|(\tilde{N} g)^{n} / \tilde{N}_{j}\right\| \cdot\left\|(T g)^{-n} /(T g)^{n} T_{i}\right\| \leqslant C^{\prime} \lambda^{n}
$$

for all $n \in \mathbf{Z}^{+}$or

$$
\left\|(\tilde{N} g)^{-n} /(\tilde{N} g)^{n} \tilde{N}_{j}\right\| \cdot\left\|(T g)^{n} / T_{i}\right\|<C^{\prime} \lambda^{n}
$$

for all $n \in \mathbf{Z}^{+}$.

Proof. Suppose that $(\tilde{N} g)^{m} / \tilde{N}_{j}<(T g)^{m} / T_{i}$ for all $i<i_{0}$ and $(T g)^{m} / T_{i}<$ $(\tilde{N} g)^{m} N_{j}$ if $i^{\prime}>i_{0}$. We claim that

$$
\begin{gathered}
\left\|(\tilde{N} g)^{n} / \tilde{N_{j}}\right\| \cdot\left\|(T g)^{-n} /(T g)^{n} T_{-}\right\| \leqslant C^{\prime} \lambda^{n}, \\
\left\|(\tilde{N} g)^{-n} /(\tilde{N} g)^{n} N_{j}\right\| \cdot\left\|(T g)^{n} / T_{+}\right\|<C^{\prime} \lambda^{n}
\end{gathered}
$$

for all $n \in \mathbf{Z}^{+}$, where $T^{+}=T_{1} \oplus \cdots \oplus T_{i_{0}}, T^{-}=T_{i_{0}+1} \oplus \cdots \oplus T_{k}$, where $C^{\prime}>0$ is a constant independent of $g \in \mathcal{S}, x \in \operatorname{Per}(g)$ and $n \in \mathbf{Z}^{+}$ and $\lambda$ satisfies (14) and (15). If we prove this claim we are done. Let us prove $\left(27^{\prime}\right)\left(\left(27^{\prime \prime}\right)\right.$ follows in a similar way). Take $v \in \tilde{N}_{j}, w \in T^{\prime}$ with $\|w\|=1$. Let $\Re$ be an orthonormal basis of $T_{-}$containing $w$. Define a linear map $L$ : $T^{-} \rightarrow \tilde{N}_{j}$ by $L u=v$ if $u \in \mathscr{B}$ and $L u=0$ if $u \in T_{+}$. There exists a constant $\gamma^{\prime}>0$ depending only on $\gamma$ such that $\|L\|<\gamma^{\prime}\left\|L / T^{-}\right\|$. Hence $\|L\|<$ $\gamma^{\prime}\|v\|$. Clearly $L \in E_{x}^{s}(g)$. Therefore, by (14),

$$
\left\|(\tilde{N} g)^{n} v\right\| /\left\|(\tilde{N} g)^{n} w\right\| \leqslant\left\|\Phi_{g}^{n}(L)\right\|<C \lambda^{n}\|L\|<C \gamma^{\prime} \lambda^{n}\|v\| .
$$

Since 


$$
\begin{aligned}
& \left\|(\tilde{N} g)^{n} / \tilde{N}{ }_{j}\right\| \cdot\left\|(T g)^{-n} /(T g)^{n} T^{-}\right\| \\
& \quad=\sup \left\{\frac{\left\|(\tilde{N} g)^{n} v\right\|}{\|v\| \cdot\left\|(T g)^{n} w\right\|} \mid v \in \tilde{N}_{j}, w \in T^{-},\|w\|=1\right\},
\end{aligned}
$$

putting $C^{\prime}=C \gamma^{\prime},\left(27^{\prime}\right)$ follows from (28).

Finally we want to prove that if $g \in \mathcal{S}$ and $x$ is a periodic point of $g / V$ with period $m$ there exists a subspace $N_{x} \subset T_{x} M$ that is a $(T g)^{m}$-invariant complement of $T_{x} V$, and we shall give an upper bound for $\alpha\left(\left(T_{x} V\right)^{\perp}, N_{x}\right)$. For this let us say that if $g \in \mathcal{S}$ and $x \in \operatorname{Per}(g)$, the multindex $\sigma=$ $\left(n_{1}, \ldots, n_{k}, m_{1}, \ldots, m_{l}\right)$ is the signature of $x$ if the canonical splittings at $x$ are (24) and (25) and satisfy $\operatorname{dim} \tilde{N}_{i}=n_{i}, \operatorname{dim} T_{j}=m_{j}$ for all $1<i \leqslant k$, $1 \leqslant j \leqslant l$. Let $\Lambda_{\sigma}(g)$ be the set of periodic points of $g / V$ with signature $\sigma$ and let $\bar{\Lambda}_{\sigma}(g)$ be its closure. By Proposition 1.2 and Lemma 2.6 there exist splittings $\tilde{N} V / \bar{\Lambda}_{\sigma}(g)=\tilde{N}_{1} \oplus \cdots \oplus \tilde{N}_{l}, \quad T V / \bar{\Lambda}_{\sigma}(g)=T_{1} \oplus \cdots \oplus T_{k}$ satisfying

$$
\tilde{N} g / \tilde{N}_{i^{\prime}}>\tilde{N} g / \tilde{N}_{i^{\prime \prime}}, \quad T g / T_{j^{\prime}}>T g / T_{j^{\prime \prime}}
$$

for all $1 \leqslant i^{\prime} \leqslant i^{\prime \prime} \leqslant l, 1 \leqslant j^{\prime}<j^{\prime \prime} \leqslant k$ and $\tilde{N} g / \tilde{N}_{i}>T g / T_{j}$ or $T g / T_{j}>$ $\tilde{N} g / \tilde{N}_{i}$ for all $1 \leqslant i \leqslant l, 1 \leqslant j \leqslant k$. By Proposition 1.1 there exist continuous $T f$-invariant subbundles $N_{1}, \ldots, N_{l}$ of $T M / \bar{\Lambda}_{\sigma}(g)$ such that

$$
T_{1} \oplus \cdots \oplus T_{k} \oplus N_{1} \oplus \cdots \oplus N_{l}=T M / \bar{\Lambda}_{\sigma}(g) .
$$

By Lemma 2.6 and Proposition 1.1 there exists $\gamma_{1}>0$ depending on $\gamma$ (given by Lemma 2.5 ), $C^{\prime}$ and $\lambda$ (given by 2.6 ) such that

$$
\alpha\left(\left(T_{x} V\right)^{\perp},\left(N_{1} \oplus \cdots \oplus N_{l}\right)_{x}\right)<\gamma_{1}
$$

for all $x \in \bar{\Lambda}_{\boldsymbol{o}}(g)$. Resuming we have proved the following property:

LEMMA 2.7. There exists $\gamma_{1}>0$ such that for all $g \in \mathcal{S}$ and every signature $\sigma$, there exists a continuous Tf-invariant subbundle $N$ of $T M / \bar{\Lambda}_{\sigma}(g)$ such that

$$
T V / \bar{\Lambda}_{\sigma}(g) \oplus N=T M / \bar{\Lambda}_{\sigma}(g), \quad \alpha\left(\left(T_{x} V\right)^{\perp}, N_{x}\right) \leqslant \gamma_{1}
$$

for all $x \in \bar{\Lambda}_{\sigma}(g)$.

Let us return now to the situation we were considering before Lemma 2.5: we have $g_{0} \in \mathcal{S}$ near to $f$ such that $\operatorname{Per}\left(g_{0}\right)$ contains a subset $W$, open in the relative topology in $\operatorname{Per}\left(g_{0}\right)$ such that $\tilde{N}_{x}^{s}\left(g_{0}\right) \cap \tilde{N}_{x}^{u}\left(g_{0}\right) \neq\{0\}$ for all $x \in W$. Since the set of possible signatures is finite, for some $\sigma$ the set $W \cap \Lambda_{\sigma}(g)$ contains an open subset $W_{0}$ of $\operatorname{Per}\left(g_{0}\right)$. Let $\gamma_{1}$ be the constant given in Lemma 2.7. Take $\beta>0$ and $\rho>0$ satisfying the following property: If $x \in V, S \subset T_{x} M$ is a suspace satisfying $S \oplus T_{x} V=T_{x} M, \alpha\left(\left(T_{x} V\right)^{\perp}, S\right) \leqslant$ $2 \gamma_{1}, v \in S, y \in V, w \in N_{y} V$ and $\|v\| \leqslant \rho,\|w\| \leqslant \rho$ and $\exp (v)=\exp (w)$ :

$$
\|x-y\| \leqslant \beta\|v\|, \quad\|v\| \leqslant \beta\|w\| .
$$

Let $\delta=\min \left(\rho, \frac{2}{3} \beta\right)$. Since $g_{0}$ is near to $f$ we can suppose that $g_{0} \in \mathcal{Q}_{\delta / 2}$ 
(see Definition 2.1). Using $g_{0}$ we shall construct $\bar{g} \in \mathcal{Q}_{2 \delta / 3}$ such that $V_{\bar{g}}=$ $\{\exp h(p) / p \in V\}$ where $h$ is a section of $N V$ with $\operatorname{Lip}(h)>\delta$, contradicting $\bar{g} \in \mathcal{Q}_{2 \delta / 3}$. For this take the subbundle $N$ of $T M / \bar{\Lambda}_{\sigma}\left(g_{0}\right)$ given by Lemma 2.7. There exist a neighborhood $B$ of $\bar{\Lambda}_{\sigma}(g)$ in $M$, a $C^{\infty}$ subbundle $N^{\prime}$ of $T M / B$ satisfying $N^{\prime} / \bar{\Lambda}_{o}\left(g_{0}\right) \oplus T V / \bar{\Lambda}_{o}\left(g_{0}\right)=T M / \bar{\Lambda}_{\sigma}\left(g_{0}\right), \alpha\left(\left(T_{x} V\right)^{\perp}, N_{x}^{\prime}\right)$ $\leqslant 2 \gamma_{2}$ for all $x \in \bar{\Lambda}_{o}\left(g_{0}\right)$ and a diffeomorphism $g \in \mathcal{Q}_{3 \delta / 5}$ such that $g \in \mathcal{\delta}$, $g / V=g_{0}, N^{\prime} / \bar{\Lambda}_{\sigma}\left(g_{0}\right)$ is $T g$-invariant, $\tilde{N} g=\tilde{N} g_{0}$ and $g(\exp v)=\exp ((T g) v)$ for all $v \in N^{\prime} / \bar{\Lambda}_{\sigma}\left(g_{0}\right)$ small enough, say with $\|v\| \leqslant r$. Denote $\Lambda=\bar{\Lambda}_{\sigma}\left(g_{0}\right)$. We start the construction of $\bar{g}$ proving the following property:

LeMma 2.8. For all $0 \neq v \in N^{\prime} / \Lambda$ the set $\left\{\left\|(T g)^{n} v\right\| \mid n \in \mathbf{Z}\right\}$ is unbounded.

Proof. Suppose that $0 \neq v \in N^{\prime} / \Lambda$ and $\left\{\left\|(T g)^{n} v\right\| \mid n \in \mathbf{Z}\right\}$ is bounded, say by $K$. Take $w=\lambda v$ such that $K \lambda \leqslant r / 2$. Then $d\left(g^{n}(\exp w), V\right)=$ $d\left(\exp (T g)^{n} w, V\right) \leqslant K \lambda$ for all $n \in \mathbf{Z}^{+}$, hence $g^{n}(\exp w) \in \bigcap_{n \in \mathbf{Z}} g^{n}(U)$ if $\lambda$ is small enough (where $U$ is given by Definition 2.1). Therefore $V_{g} \supset\{w\} \cup$ $V$, contradicting Definition 2.1.

By the results in [13] or [21] this lemma implies that there exists a continuous $T f$-invariant splitting $N^{\prime} / \Lambda=N^{s} \oplus N^{u}$ and constants $C>0$, $0<\sigma<1$ such that

$$
\left\|(T g)^{n} / N_{x}^{s}\right\| \leqslant C \sigma^{n}, \quad\left\|(T g)^{-n} / N_{x}^{u}\right\| \leqslant C \sigma^{n}
$$

for all $n \in \mathbf{Z}^{+}$. Take $x_{0} \in W_{0}$. The condition $\tilde{N}_{x_{0}}^{s}(g) \cap \tilde{N}_{x_{0}}^{u}(g) \neq\{0\}$ implies that there exists an eigenvalue $\lambda$ of $(\tilde{N} g)^{m} / \tilde{N}_{x_{0}} V\left(m\right.$ being the period of $\left.x_{0}\right)$ and eigenvalues $\lambda_{1}, \lambda_{2}$ of $(T g)^{m} / T_{x} V$ such that $\left|\lambda_{1}\right|<|\lambda|<\left|\lambda_{2}\right|$. Otherwise $E_{x}^{s}(g)$ (resp. $E_{x}^{u}(g)$ ) would be the space of linear maps from $T_{x} V$ in the subspace $\tilde{N}^{+}\left(\tilde{N}^{-}\right)$of $\tilde{N}_{x} V$ spanned by the invariant subspaces associated to eigenvalues with modulus smaller (greater) than the modulus of every eigenvalue of $(T g)^{m} / T_{x} V$; hence $\tilde{N}_{x_{0}}^{s}(g)=\tilde{N}^{+}, \tilde{N}_{x_{0}}^{u}(g)=\tilde{N}^{-}$. Since every period of $g$ is hyperbolic (because $g \in \mathcal{S}$ ) there exist two possible cases: $\left|\lambda_{1}\right|<|\lambda|<$ $\left|\lambda_{2}\right|$ and $1<|\lambda|<\left|\lambda_{2}\right|$. Consider the case $\left|\lambda_{1}\right|<|\lambda|<1$. The other case is handled with the same method. Let $N_{0} \subset N_{x_{0}}^{\prime}$ be the subspace associated to the eigenvalue $\lambda$, and $T_{0} \subset T_{x_{0}} V$ be the subspace associated with $\lambda_{1}$. There exists an embedded disc $D \subset V$ containing $x_{0}$ and satisfying $T_{x_{0}} D=T_{0}$, $g(D) \subset D$. We have two possible situations: either every neighborhood of $x_{0}$ in $D$ contains a wandering point of $g_{0} / V$ or it does not. Suppose that we have the second situation. Then $\Lambda \cap D$ is a neighborhood of $x_{0}$ in $D$. Take $y \in \Lambda \cap D$. By [9] there exists a continuous subbundle $N_{0}^{\prime}$ of $N^{\prime} / D$ such that $(T g)^{m} N_{0}^{\prime} \subset N_{0}^{\prime}$ and $N_{0, x_{0}}^{\prime}=N_{0}$. The properties of $D$ imply $\lim \left\|(T g)^{n} / N_{0, x_{0}}^{\prime}\right\|$ $=0$ for all $x \in D$. Hence $N_{0}^{\prime} \subset N^{s} / D$. Take a $C^{\infty}$ section $\xi$ of $T M$ such that $\xi / B$ is a section of $N^{\prime}$, the support of $\xi$ is contained in a neighborhood $U_{0} \subset \Lambda$ of a point $y \in D$ satisfying $x_{0} \notin U_{0}, \xi(y) \neq 0, \cup_{1}^{k} g^{j}\left(U_{0}\right) \cap U_{0}=\varnothing$, where $k$ satisfies $\sigma^{k+1} r \leqslant 2\|\xi(y)\|$. Define a section $\eta$ of $N^{\prime} V$ by 


$$
\eta(x)=\sum_{0}^{\infty}(T g)^{n} \xi\left(g^{-n}(x)\right) .
$$

For all $x \in \Lambda$ we have

$$
\|\eta(x)\| \leqslant \sum_{0}^{\infty}\left\|(T g)^{n} \xi\left(g^{-n}(x)\right)\right\| \leqslant C \sum_{0}^{\infty} \sigma^{n}\left\|\left((T g)^{-n}(x)\right)\right\| .
$$

Suppose that $\|\xi(p)\| \leqslant r(1-\sigma) / 2 C$ for all $p \in V$. Then

$$
\|\eta(x)\| \leqslant r / 2
$$

for all $x \in V$. The condition $g^{j}\left(U_{0}\right) \cap U_{0}=\varnothing$ for all $1<j<k$ implies

$$
\begin{aligned}
\|\eta(y)\| & >\|\xi(y)\|-\sum_{k+1}^{\infty}\left\|(T g)^{n} \xi\left(g^{-n}(y)\right)\right\| \\
& >\|\xi(y)\|-C \sigma^{k+1} /(1-\sigma) \cdot r(1-\sigma) / 2 C>0
\end{aligned}
$$

and it follows from the definition of $\eta$ that

$$
(T g) \eta(x)+\xi(g(x))=\eta(g(x))
$$

for all $x \in \Lambda$. Given $\mu \in R$ take $\bar{g}$ such that

$$
\begin{gathered}
\bar{g} \in \mathscr{U}_{\delta}, \\
\bar{g}(\exp v)=\exp \left(\mu \xi(g(x))+\left(T_{g}\right) v\right)
\end{gathered}
$$

for all $x \in \Lambda, v \in N_{x}^{\prime}$ with $\|v\| \leqslant \mu r / 2$. If $|\mu|$ is small there exists $\bar{g}$ satisfying (32) and (33). By (33) and (31) we have

$$
\bar{g}(\exp \mu \eta(x))=\exp (\mu \xi(g(x))+(T g) \eta(x))=\exp \mu \eta(g(x)) .
$$

Hence,

$$
\bar{g}^{n}(\exp \mu \eta(x))=\exp \mu \eta\left(g^{n}(x)\right)
$$

for all $n \in \mathbf{Z}, x \in \Lambda$. This and (29) imply that the distance between $\bar{g}^{n}(\exp \mu \eta(x))$ and $V$ is $\leqslant \mu r / 2$ for all $n \in \mathbf{Z}$. Therefore, by Definition 2.1, this proves that $\exp \mu \eta(x) \in V_{\bar{g}}$ for all $x \in \Lambda$. Now take $\left|\lambda_{1}\right|<c_{1}<c<|\lambda|$ and $K>0$ satisfying $\left\|(T g)^{n} v\right\|>K c^{n}\|v\|$ for all $n \in \mathbf{Z}^{+}, v \in N_{0, y}^{\prime}$ and $\left\|g^{n}(y)-g^{n}\left(x_{0}\right)\right\| \leqslant K c_{1}^{n}$ for all $n \in \mathbf{Z}^{+}$. By the condition $g^{n}(y) \notin U_{0}$ for all $n>1$ and (30),

$$
\eta\left(g^{n}(y)\right)=(T g)^{n} \eta(y) .
$$

Hence

$$
\frac{\left\|\eta\left(g^{n}(y)\right)\right\|}{\left\|g^{n}(y)-g^{n}\left(x_{0}\right)\right\|}=\frac{\left\|(T g)^{n} \eta(y)\right\|}{\left\|g^{n}(y)-g^{n}\left(x_{0}\right)\right\|} \geqslant\left(\frac{c}{c_{1}}\right)^{n}\|\eta(y)\| .
$$

Now let $h \in \Gamma_{\mathfrak{R}}(N V)$ such that $V_{\bar{g}}=\{\exp h(p) / p \in V\}$ and $\|h\|_{\mathfrak{R}}<\delta$. We claim that $\exp \mu \eta(y) \in V_{\bar{g}}$. This follows from (34), which gives $\bar{g}^{n}(\exp \mu \eta(y))$ $=\exp \mu \eta\left(g^{n}(y)\right)$ for all $n \in \mathbf{Z}$. Hence if $\mu$ is small enough, $\bar{g}^{n}(\exp \mu \eta(y)) \in$ $U$ ( $U$ given by Definition 2.1); thus $\exp \mu \eta(y) \in V_{\bar{g}}$. Take $z_{n} \in V$ such that 
$\exp h\left(z_{n}\right)=\bar{g}^{n}(\exp \mu \eta(y))=\exp \mu \eta\left(g^{n}(y)\right)$. Observing that the $g$-orbit and $\bar{g}$-orbit of $x_{0}$ are the same we obtain $h\left(g^{n}\left(x_{0}\right)\right)=0$ for all $n \in \mathbf{Z}$. Therefore,

$$
\begin{aligned}
\operatorname{Lip}(\eta) & \geqslant \frac{\left\|h\left(z_{n}\right)-h\left(g^{n}\left(x_{0}\right)\right)\right\|}{\left\|z_{n}-g^{n}\left(x_{0}\right)\right\|}=\frac{\left\|h\left(z_{n}\right)\right\|}{\left\|z_{n}-g_{n}\left(x_{0}\right)\right\|} \\
& \geqslant \frac{1}{\left\|z_{n}-g^{n}(y)\right\| /\left\|h\left(z_{n}\right)\right\|+\left\|g^{n}(y)-g^{n}\left(x_{0}\right)\right\| /\left\|h\left(z_{n}\right)\right\|} \\
& \geqslant \frac{1}{\beta\left(1+\left\|g^{n}(y)-g^{n}\left(x_{0}\right)\right\| /\left\|h\left(z_{n}\right)\right\|\right)} \geqslant \frac{1}{\beta\left(1+\left(c_{1} / c\right)^{n} / \mu\|\eta(y)\|\right)} .
\end{aligned}
$$

Since by (30) $\|\eta(y)\|>0$, taking $n \rightarrow+\infty$ we obtain $\operatorname{Lip}(\eta)>1 / 2 \beta=\delta$, contradicting (32). This completes the proof when $x_{0}$ is in the closure of $\operatorname{Per}\left(g_{0}\right)-\left\{x_{0}\right\}$.

It remains to consider the case when $\Omega(g / V) \cap D$ is not a neighborhood of $x_{0}$ in $D$. This case is considered in the next lemma.

LEMMA 2.8. There exists a neighborhood $\mathfrak{V}$ of $f$ such that if $g \in \mathfrak{V} \cap \mathcal{\delta}$, $x \in \operatorname{Per}(g)$ and $m$ is the period of $x$, then for every eigenvalue $\lambda_{1}$ of $(T g)^{m} / T_{x} V$ and every eigenvalue $\lambda$ of $(\tilde{N} g)^{m} / N_{x} V$ if we have $\left|\lambda_{1}\right|\left\langle|\lambda|\left\langle 1\left(\left|\lambda_{1}\right|\right\rangle|\lambda|\right\rangle\right.$ 1), then every embedded disc $D \subset V$ such that $g^{m}(D) \subset D\left(g^{-m}(D) \subset D\right)$ and $T_{x} D$ is the subspace of $T_{x} V$ associated to $\lambda_{1}$, is contained in $\Omega(g / V)$.

PRoof. If the lemma is false we can find $g \in Q_{\delta}$, where $\delta=1 / 2 \beta$ ( $\beta$ and $\rho$ satisfying the same properties as in the previous proof), and $x \in \operatorname{Per}(g)$ with an eigenvalue $\lambda_{1}$ of $(T g)^{m} / T_{x} V(m$ the period of $x)$ and an eigenvalue $\lambda$ of $(\tilde{N} g)^{m} / \tilde{N}_{x} V$ such that, for instance, $\left|\lambda_{1}\right|<|\lambda|<1$ and there exists an embedded disc $D \subset V$ satisfying $g^{m}(D), T_{x} D$ is the subspace associated to the eigenvalue $\lambda_{1}$ and $D-\Omega(g / V) \neq \varnothing$. Take $y \in D-\Omega(g / V)$, a neighborhood $U_{0}$ of $y$ satisfying $g^{n}\left(U_{0} \cap V\right) \cap U_{0} \neq \varnothing$ for all $n \in \mathbf{Z}$ and a disc $D_{0} \subset M$ containing $y$ such that $\lim _{n \rightarrow+\infty}\left\|g^{n}(z)-g^{n}(y)\right\|=0$ for all $z \in D_{0}$ and there exist $k>0,\left|\lambda_{1}\right|<c<|\lambda|$ satisfying

$$
\left\|g^{n}(z)-g^{n}(y)\right\| \geqslant K c^{n}\|z-y\|
$$

for all $n \in \mathbf{Z}, z \in D_{0}$. Moreover, if $\left|\lambda_{1}\right|<c_{1}<c$ there exist $K_{1}>0$, such that

$$
\left\|g^{n}(z)-g^{n}(x)\right\| \leqslant K_{1} c_{1}^{n}\|z-x\|
$$

for all $z \in D, n \in \mathbf{Z}^{+}$. Choose $\bar{g} \in \mathcal{Q}_{\delta}$ with $\bar{g}\left(g^{-1}(y)\right)=\bar{y} \in D_{0}-V, \bar{y} \neq y$ and $g(p)=\bar{g}(p)$ for all $p \notin g^{-1}\left(U_{0}\right)$.

The condition $g^{n}\left(U_{0}\right) \cap U_{0}=\varnothing$ for all $n \in \mathbf{Z}$ implies that $\left\{g^{n}(\bar{y}) \mid n \in\right.$ $\left.\mathbf{Z}^{+}\right\} \cup\left\{g^{-n}\left(g^{-1}(y)\right) \mid n \in \mathbf{Z}^{+}\right\}$is a $\bar{g}$-orbit. Hence $\bar{y} \in V_{g}$. Let $h \in \Gamma_{\mathbb{Q}}(N V)$ satisfy $V_{\bar{g}}=\{\exp h(p) \mid p \in V\}$. Let $z_{n} \in V$ be such that $h\left(z_{n}\right)=g^{n}(\bar{y})$. Using (35), (36) as in the previous proof, we conclude $\|h\|_{\mathcal{L}}>1 / 2 \beta$, contradicting $g \in \mathcal{Q}_{\delta}$.

Proof of Proposition 2.1. Define $N_{x}^{s} V\left(N_{x}^{u} V\right)$ as the set of $v \in T_{x} M$ such 
that $\lim \left\|(T f)^{n} V\right\| \cdot\left\|(T)^{-n} / T_{f^{n}(x)} V\right\|=0 \quad\left(\lim \left\|(T f)^{-n} v\right\| \cdot\left\|(T f)^{n} / T_{f^{-n}(x)} V\right\|\right.$ =0). Observe that when $x \in \Omega(f / V), N_{x}^{s} V, N_{x}^{u} V$ are the fibers at $x$ of the subbundles $N^{s}, N^{u}$ of $T M / \Omega(f / V)$ given by Lemma 2.3. We want to prove that the maps $x \rightarrow N_{x}^{s} V, x \rightarrow N_{x}^{u} V$ define continuous subbundles of $T M / V$ satisfying all the conditions required by the definition of normal hyperbolicity. Clearly $(T f) N_{x}^{s} V=N_{f(x)}^{s} V,(T f) N_{x}^{u} V=N_{f(x)}^{u} V$ for all $x \in V$. Let us prove that for all $x \in V, N_{x}^{s} V \cap N_{x}^{u} V=\{0\}$. Let $\pi: T M / V \rightarrow \tilde{N} V$ be the canonical projection. Let $v \in N_{x}^{s} V \cap N_{x}^{u} V$ and $\tilde{v}=\pi v$. If $0 \neq v$ we can take a linear map $0 \neq L: T_{x} V \rightarrow\{\lambda v \mid \lambda \in \mathbf{R}\}$. Then $L \in E_{x}^{s}(f) \cap E_{x}^{u}(f)$, contradicting Lemma 2.3. Now let us show that $T_{x} M=T_{x} V \oplus N_{x}^{s} V \oplus N_{x}^{u} V$ for all $x \in V$. We start with the following proposition:

LeMma 2.9. Let $\pi_{0}: T M / V \rightarrow T V, \pi: T M / V \rightarrow N V$ be the projections associated to the splitting $T M / V=T V \oplus N V$. There exists $\delta>0$ such that if $x \in V, v \in T_{x} M$ and $\left\|\pi(T f)^{n} v\right\| \leqslant \delta\left\|\pi_{0}(T f)^{n} v\right\|$ for all $n \in \mathbf{Z}^{+}$then $v \in N_{x}^{s} V$ $\bigoplus T_{x} V$.

Proof. By Proposition 1.3 there exist a neighborhood $U$ of $\Omega(f / V)$ and a continuous subbundle $\hat{N}^{s}$ of $T V / \Lambda^{s}$, where $\Lambda^{s}=\bigcap_{n>0} f^{-n}(U)$, satisfying

$$
\begin{gathered}
(T f) \hat{N}^{s} \subset \hat{N}^{s}, \\
\hat{N}^{s} / \Omega(f / V)=N^{s} .
\end{gathered}
$$

From these relations it follows that $\hat{N}_{x}^{s} \subset N_{x}^{s} V$ for all $x \in V$ (in fact it is possible to prove $\hat{N}_{x}^{s}=N_{x}^{s} V$, but we shall not need this property). Moreover, we can assume that there exists a continuous subbundle $\hat{N}^{u}$ of $T M / \Lambda^{s}$ such that $\hat{N}^{u} / \Omega(f / V)=N^{u}$ and $\hat{N}^{u} \oplus \hat{N}^{s} \oplus T V / \Lambda^{s}=T M / \Lambda^{s}$. Let $\pi^{s}: T M / \Lambda^{s}$ $\rightarrow \hat{N}^{s}, \pi^{u}: T M / \Lambda^{s} \rightarrow \hat{N}^{u}, \pi^{c}: T M / \Lambda^{s} \rightarrow T V / \Lambda^{s}$ be the projections associated to this splitting. Define $\pi^{c s}=\pi^{c}+\pi^{s}$ and for $x \in \Lambda^{s}, \varepsilon>0$ define the cones

$$
S_{\varepsilon}(x)=\left\{v \in T_{x} M /\left\|\pi^{c s} v\right\| \leqslant \varepsilon\left\|\pi^{u} v\right\|\right\} .
$$

There exist $m \in \mathbf{Z}^{+}, \varepsilon>0$ satisfying

$$
\begin{gathered}
(T f) S_{\varepsilon}(x) \subset S_{\varepsilon / 2}(f(x)), \\
\left\|(T f)^{m} v\right\| /\left\|(T f)^{m} w\right\| \geqslant 2\|v\| /\|w\|
\end{gathered}
$$

for all $x \in \Omega(f / V), v \in S_{\varepsilon}(x), 0 \neq w \in N_{x}^{s} \oplus T V$. Let $U_{0} \subset U$ be a neighborhood of $\Omega(f / V)$ such that if $\Lambda_{0}^{s}=\bigcap_{n>0} f^{-n}\left(U_{0}\right)$ then these relations remain true for all $x \in \Lambda_{0}^{s}, v \in S_{\varepsilon}(x), 0 \neq w \in N_{x}^{s} \oplus T_{x} V$. From (37) and (38) it follows that there exist $C>0,0<\sigma<1$ satisfying

$$
\left\|(T f)^{n} v\right\| /\left\|(T f)^{n} w\right\| \geqslant C(1 / \sigma)^{n}\|v\| /\|w\|
$$

for all $x \in \Lambda_{0}^{s}, v \in S_{\varepsilon}(x), 0 \neq w \in \hat{N}_{x}^{s} \oplus T_{x} V$. Take $\delta>0$ such that $v \notin$ $S_{\varepsilon}(x)$ for all $x \in V$ and $v \in T_{x} M$ such that $\|\pi v\| \leqslant \delta\left\|\pi_{0} v\right\|$. Now suppose that $y \in V, v \in T_{y} M$ satisfy $\left\|\pi(T f)^{n} v\right\| \leqslant \delta\left\|\pi_{0}(T f)^{n} v\right\|$ for all $n \in \mathbf{Z}^{+}$. We 
want to prove $v \in N_{y}^{s} V \oplus T_{y} V$. Suppose $v \notin N_{y}^{s} V \oplus T_{y} V$. Hence if $f^{n_{1}}(y)=$ $x \in \Lambda_{0}^{s}$ we have $(T f)^{n} v \notin N_{x}^{s} V \oplus T_{x} V$. Write $(T f)^{n} v=v^{\prime}+v^{\prime \prime}$ where $v^{\prime} \in$ $\hat{N}_{x}^{u}, v^{\prime \prime} \in N_{x}^{s} \oplus T_{x} V, v^{\prime} \neq 0$. By (39) we have

$$
\left\|(T f)^{n} v^{\prime}\right\| /\left\|(T f)^{n} v^{\prime \prime}\right\|>C(1 / \sigma)^{n}\left\|v^{\prime}\right\| /\left\|v^{\prime \prime}\right\|
$$

for all $n \in \mathbf{Z}^{+}$. Then

$$
\left\|\pi^{c s}(T f)^{n+n_{1}} v\right\|=\left\|\pi^{c s}(T f)^{n}\left(v^{\prime}+v^{\prime \prime}\right)\right\| \leqslant\left\|\pi^{c s}(T f)^{n} v^{\prime}\right\|+\left\|(T f)^{n} v^{\prime \prime}\right\| .
$$

Since $v^{\prime} \in \hat{N}_{x}^{u} \subset S_{\varepsilon}$, we obtain

$$
\left\|\pi^{c s}(T f)^{n} v^{\prime}\right\| \leqslant \varepsilon\left\|\pi^{u}(T f)^{n} v^{\prime}\right\|=\varepsilon\left\|\pi^{u}(T f)^{n}\left(v^{\prime}+v^{\prime \prime}\right)\right\|=\varepsilon\left\|\pi^{u}(T f)^{n+n_{1}} v\right\| ;
$$

hence,

$$
\left\|\pi^{c s}(T f)^{n+n_{1}} v\right\| \leq\left(\varepsilon+\frac{\left\|(T f)^{n} v^{\prime \prime}\right\|}{\left\|\pi^{u}(T f)^{n+n_{1}} v\right\|}\right)\left\|\pi^{u}(T f)^{n+n_{1}} v\right\| .
$$

This inequality, together with (40), gives

$$
\left\|\pi^{c s}(T f)^{n+n_{1}} v\right\|<\left(\varepsilon+\frac{\sigma^{n}\left\|v^{\prime \prime}\right\|}{C\left\|v^{\prime}\right\|}\right)\left\|\pi^{u}(T f)^{n+n_{1}} v\right\| .
$$

Hence $(T f)^{n+n_{w}} \in S_{2 \varepsilon}(x)$ for large values of $n$, thus contradicting the definition of $\delta$.

Using this lemma we shall prove

$$
N_{x}^{s} V+N_{x}^{u} V+T_{x} V=T_{x} M
$$

for all $x \in V$. Together with the property $N_{x}^{s} V \cap N_{x}^{u} V=\{0\}$, this relation proves

$$
N_{x}^{s} V \oplus N_{x}^{u} V \oplus T_{x} V=T_{x} M
$$

for all $x \in V$. Suppose that for some $x$ we have $N_{x}^{s} V+N_{x}^{u} V+T_{x} V \neq T_{x} M$. By Lemma 2.4, $x \notin \Omega(f / V)$. Let $\delta>0$ be the number given by Lemma 2.9 and $U_{\delta}$ the neighborhood of $f$ given by Definition 2.1. Let $W$ be a neighborhood of $x$ such that $f^{n}(W) \cap W=\varnothing$ for all $n \in \mathbf{Z}$ and let $g \in Q_{\delta}$ be a diffeomorphism satisfying $g(y)=f(y)$ for all $y$ in a neighborhood of $V-W$, $g(x)=f(x), g\left(f^{-1}(x)\right)=x$ and

$$
\operatorname{dim}\left((T g)\left(N_{f^{-1}(x)}^{u} V \oplus T_{f^{-1}(x)} V\right) \cap\left(N_{x}^{s} V \oplus T_{x} V\right)\right)<\operatorname{dim} V
$$

let $h \in T_{\mathrm{e}}(N V)$ such that $V_{g}=\{\exp h(p) \mid p \in V\}$. Since $h \in \Gamma_{\mathfrak{B}}$ given any basis $\left\{v_{1}, \ldots, v_{l}\right\}$ of $T_{x} V$, there exist $\left\{w_{1}, \ldots, w_{l}\right\} \subset N_{x} V$ such that

$$
\liminf _{\lambda \rightarrow 0}\left\|\frac{h\left(\exp \lambda v_{i}\right)-h(x)}{\lambda}-w_{i}\right\|=0
$$

for $1<i \leqslant l$, where exp: $T V \rightarrow V$ is the exponential mapping of the Riemann manifold $V$. The condition $\|h\|_{\mathcal{L}}<\delta$ implies $\left\|w_{i}\right\| /\left\|v_{i}\right\|<\delta$ for all $1<i<l$. For all $n \in \mathbf{Z}^{+}$, by the invariance of $V_{g}$, we have 


$$
\liminf _{\lambda \rightarrow 0}\left\|\frac{h\left(\exp \lambda \pi_{0}(T g)^{n}\left(v_{i}+w_{i}\right)\right)-h\left(g^{n}(x)\right)}{\lambda}-\pi(T f)^{n}\left(v_{i}+w_{i}\right)\right\|=0 .
$$

Since $\|h\|_{\mathcal{L}}<\delta$ we obtain

$$
\left\|\pi(T g)^{n}\left(v_{i}+w_{i}\right)\right\|<\delta\left\|\pi_{0}(T g)^{n}\left(v_{i}+w_{i}\right)\right\| .
$$

But $g$ satisfies $(T g)^{n} /\left(T_{f(x)} M\right)=(T f)^{n}$ and $(T g)^{-n} / T_{x} M=(T f)^{-n} / T_{x} M$ for all $n \in \mathbf{Z}^{+}$. Therefore, by Lemma 2.9,

$$
\begin{gathered}
\left\{(T g)\left(v_{i}+w_{i}\right) \mid i=1, \ldots, l\right\} \subset N_{f(x)}^{s} V \oplus T_{f(x)} V, \\
\left\{v_{i}+w_{i} \mid i=1, \ldots, l\right\} \subset N_{x}^{u} V \oplus T_{x} V .
\end{gathered}
$$

Let $S$ be the space spanned by $\left\{v_{i}+w_{i} \mid i=1, \ldots, l\right\}$. Then

$$
\operatorname{dim}\left((T g)\left(N_{x}^{u} V \oplus T_{x} V\right) \cap\left(N_{x}^{s} V \oplus T_{x} V\right)\right)>\operatorname{dim} S=\operatorname{dim} V,
$$

contradicting (41).

Now we know that $N_{x}^{s} V \oplus N_{x}^{u} V \oplus T_{x} V=T_{x} M$ for all $x \in V$. Our next step is to show that the maps $x \rightarrow N_{x}^{s} V, x \rightarrow N_{x}^{u} V$ define continuous subbundles of $T M / V$. For this define, as before, $G_{x}^{s}\left(G_{x}^{u}\right)$ as the set of linear maps $L$ : $T_{x} V \rightarrow \pi\left(N_{x}^{s} V\right)\left(L: T_{x} V \rightarrow \pi\left(N_{x}^{u} V\right)\right)$. It is clear that

$$
G_{x}^{s} \oplus G_{x}^{u}=L_{x}(V)
$$

for all $x \in V$ and

$$
\begin{aligned}
& G_{x}^{s}=E_{x}^{s}(f), \\
& G_{x}^{u}=E_{x}^{u}(f),
\end{aligned}
$$

Then $\Phi: L_{x}(V) \hookleftarrow$ (defined as in the proof of Lemma 2.3) is a hyperbolic vector bundle isomorphism and the maps $x \rightarrow E_{x}^{s}(f), x \rightarrow E_{x}^{u}(f)$ define continuous subbundles of $L_{x}(V)$ [13, Proposition 1.1]. From this and (42'), (42") it follows that the maps $x \rightarrow \pi\left(N_{x}^{s} V\right), x \rightarrow \pi\left(N_{x}^{u} V\right)$ define a continuous $\tilde{N}$-invariant splitting $\tilde{N} V=\tilde{N}^{s} V \oplus \tilde{N}^{u} V$ satisfying

$$
\tilde{N} f / \tilde{N}^{s} V>T f / T V>\tilde{N} f / N^{u} V .
$$

From Proposition 1.2 it follows that there exist continuous subbundles $N^{s} V$, $N^{u} V$ invariant under $T f$ and satisfying $\pi\left(N^{s} V\right)=\tilde{N}^{s} V, \pi\left(N^{u} V\right)=\tilde{N}^{u} V$ and

$$
T f / N^{s} V>T f / T V>T f / N^{s} V \text {. }
$$

From this it follows easily that the fibers at $x \in V$ of $N^{s} V$ and $N^{u} V$ are the spaces $N_{x}^{s} V, N_{x}^{u} V$ defined before.

It remains to prove that there exist constants $C>0,0<\lambda<1$ satisfying

$$
\begin{aligned}
\left\|(T f)^{n} / N_{x}^{s} V\right\| & <C \lambda^{n}, \\
\left\|(T f)^{-n} / N_{x}^{u} V\right\| & <C \lambda^{n}
\end{aligned}
$$

for all $n \in \mathbf{Z}^{+}, x \in V$. Let us prove (45). If we show that $\left\{\left\|(T f)^{n} v\right\| \mid n \in\right.$ $\left.\mathrm{Z}^{+}\right\}$is unbounded for all $0 \neq v \in N_{x}^{u} V, x \in \Omega(f / V)$ it follows [13, Propo- 
sition 1.1] that there exist $C_{1}>0,0<\lambda_{1}<1$ satisfying $\left\|(T f)^{-n} / N_{x}^{u} V\right\| \leqslant$ $C_{1} \lambda_{1}^{n}$ for all $x \in \Omega(f / V), n \in \mathbf{Z}^{+}$. Then it is easy to see that $\left\{\left\|(T f)^{n} v\right\| \mid n \in\right.$ $\mathrm{Z}^{+}$\} is unbounded for all $0 \neq v \in N^{u} V$. Applying again [13, Proposition 1.1], there exist $C>0,0<\lambda<1$ satisfying (45). Hence let us suppose that for some $x \in \Omega(f / V)$ there exists $0 \neq v \in N_{x}^{u} V$ such that for some $K>0$, $\left\|(T f)^{n} v\right\| \leqslant K\|v\|$ for all $n \in \mathbf{Z}^{+}$. It is not difficult to show that there exist $C_{1}>0,0<\lambda_{1}<1$, and $r>0$ satisfying

$$
\left\|f^{n}\left(x_{1}\right)-f^{n}\left(x_{2}\right)\right\| /\left\|(T f)^{n} w\right\|<C_{1} \lambda_{1}^{n}\left\|x_{1}-x_{2}\right\| /\|w\|
$$

for all $x_{i} \in V, i=1,2,0 \neq w \in N_{x_{1}}^{u} V$ and $n \in \mathbf{Z}^{+}$such that $\left\|x_{1}-x_{2}\right\|<$ $\delta(n)$, where $\delta(n)$ is so small that $\left\|f^{j}(x)-f^{j}\left(x_{2}\right)\right\| \leqslant r$ for all $0 \leqslant j \leqslant n$, if $x, x_{2}$ satisfy $\left\|x_{1}-x_{2}\right\|<\delta(n)$. Then if $y \in V$ and $\|y-x\| \leqslant \delta(n)$, we conclude that

$$
\left\|f^{n}(y)-f^{n}(x)\right\| \leqslant C_{1} \lambda_{1}^{n} \frac{\|y-x\|}{\|v\|} \cdot\left\|(T f)^{n} v\right\| \leqslant K C_{1} \lambda_{1}^{n}\|y-x\| .
$$

Take $\delta(1)>r_{1}>0$ satisfying $K C_{1} \lambda_{1}^{n} r_{1} \leqslant \delta(1)$ for all $n \in \mathbf{Z}^{+}$. Defining $\bar{K}=$ $K C_{1}$, we obtain

$$
\left\|f^{n}(y)-f^{n}(x)\right\| \leqslant \bar{K} \lambda_{1}^{n}\|y-x\|
$$

for all $n \in \mathbf{Z}^{+}$and $y \in V$ such that $\|y-x\| \leqslant r_{1}$. Since $x$ is nonwandering this implies that $x$ is periodic and there exists an eigenvalue $\lambda$ of $(T f)^{m} / N_{x}^{u} V$ ( $m$ the period of $x$ ) with $|\lambda| \leqslant 1$. Since $T f / N^{u} V>T f / T V$ it follows that $(T f)^{m} / N_{x}^{u} V>(T f)^{m} / T_{x} V$ and this proves that $|\mu|<|\lambda|$ for every eigenvalue $|\mu|$ of $(T f)^{m} / T_{x} V$, and now it is easy to approximate $f$ by $g \in \mathcal{S}$ such that $g(y)=f(y)$ for every $y$ in the orbit of $x$ and such that $(\tilde{N} g)^{m} / \tilde{N}_{x} V$ has an eigenvalue $\lambda^{\prime}$ with $\left|\lambda^{\prime}\right|<1$ such that $|\mu|<\left|\lambda^{\prime}\right|$ for all eigenvalues of $(T g)^{m} / T_{x} V$, thus contradicting Lemma 2.9 .

\section{REFERENCES}

1. J. Hadamard, Sur l'itération et les solutions asymptotiques des équations différentielles, Bull. Soc. Math. France 29 (1901), 224-228.

2. O. Perron, Uber Stabilitat und asymptotischen Verhalten der Integrals von Differentialgleichnungssystemen, Math. Z. 29 (1928), 129-160.

3. D. Ruelle and F. Takens, On the nature of turbulence, Comm. Math. Phys. 20 (1971), 167-192.

4. J. Hale, Integral manifolds of perturbed differential systems, Ann. of Math. (2) 73 (1961), 496-531.

5. S. Diliberto, Perturbation theorems for periodic surfaces. I, II, Rend. Circ. Mat. Palermo 9 (1960), 265-299; 10 (1961), 111-161.

6. R. Sacker, A perturbation theorem for invariant Riemannian manifolds, Academic Press, New York, 1967, pp. 43-54.

7. I. Kupka, Stabilité des variétés invariantes d'un champ de vecteurs pour les petites perturbations, C. R. Acad. Sci. Paris 258 (1964), 4197-4200.

8. N. Fenichel, Persistence and smoothness of invariant manifolds of flows, Indiana Univ. Math. J. 21 (1971-1972), 193-226.

9. M. Hirsch, C. Pugh and M. Shub, Invariant manifolds (to appear). 
10. R. Mañé, Variedades persitentes, Thesis, Inst. Mat. Pura Apl., Rio de Janeiro, 1973.

11. , Persistent manifolds are normally hyperbolic, Bull. Amer. Math. Soc. 80 (1974), 90-91.

12. A. Gottlieb, Converses to the $\Omega$-stability and invariant lamination theorems, Trans. Amer. Math. Soc. 202 (1975), 369-383.

13. R. Mañé, Quasi-Anosov diffeomorphisms and hyperbolic manifolds, Trans. Amer. Math. Soc. 229 (1977), 351-370.

14. C. Pugh, An improved closing lemma and a general density theorem, Amer. J. Math. 89 (1967), 1010-1021.

15. J. Franks, Necessary conditions for stability of diffeomorphisms, Trans. Amer. Math. Soc. 158 (1971), 301-308.

16. R. Mañe, Reduction of semilinear parabolic equations to finite dimensional $C^{1}$-flows (Proc. III ELAM), Lecture Notes in Math., vol. 597, Springer-Verlag, Berlin and New York, 1977, pp. 361-379.

17. J. Selgrade, Isolated invariant sets for flows on vector bundles, Trans. Amer. Math. Soc. 203 (1975), 359-390.

18. J. Kurzweil, Invariant manifolds for flows (Proc. Sympos., Universidad Puerto Rico), Academic Press, New York, 1967, pp. 431-469.

19. C. Pugh and M. Shub, Axiom A actions, Invent. Math. 29 (1975), 7-38.

instituto de Matemítica Pura e Aplicada, luiz de Camòes, 68 CEP 20000, Rio de JANEIRO, BRAZIL 